\title{
Murlentamab, a Low Fucosylated Anti-Müllerian Hormone Type II Receptor (AMHRII) Antibody, Exhibits Anti-Tumor Activity through Tumor-Associated Macrophage Reprogrammation and T Cell Activation
}

\author{
Mélissa Prat ${ }^{1, \dagger}$, Marie Salon ${ }^{1,2,+}$, Thibault Allain ${ }^{1}$, Olivier Dubreuil ${ }^{3}$, Grégory Noël ${ }^{4}$, Laurence Preisser ${ }^{5}$, \\ Bérangère Jean ${ }^{3}$, Lydie Cassard ${ }^{6}\left(\mathbb{D}\right.$, Fanny Lemée ${ }^{3}$, Isabelle Tabah-Fish ${ }^{3, \ddagger}$, Bernard Pipy ${ }^{1,2}$, Pascale Jeannin ${ }^{5}$, \\ Jean-François Prost ${ }^{3}$, Jean-Marc Barret ${ }^{3}$ (D) and Agnès Coste 1,2,*(D) $^{1}$
}

check for updates

Citation: Prat, M.; Salon, M.; Allain, T.; Dubreuil, O.; Noël, G.; Preisser, L.; Jean, B.; Cassard, L.; Lemée, F.; Tabah-Fish, I.; et al. Murlentamab, a Low Fucosylated Anti-Müllerian Hormone Type II Receptor (AMHRII) Antibody, Exhibits Anti-Tumor Activity through Tumor-Associated Macrophage Reprogrammation and T Cell Activation. Cancers 2021, 13, 1845. https://doi.org/10.3390/

cancers13081845

Academic Editor: Amir A Jazaeri

Received: 14 February 2021

Accepted: 7 April 2021

Published: 13 April 2021

Publisher's Note: MDPI stays neutra with regard to jurisdictional claims in published maps and institutional affiliations.

Copyright: (c) 2021 by the authors. Licensee MDPI, Basel, Switzerland. This article is an open access article distributed under the terms and conditions of the Creative Commons Attribution (CC BY) license (https:/ / creativecommons.org/licenses/by/ $4.0 /)$
1 UMR 152 Pharma Dev, Université de Toulouse, IRD, UPS, 31062 Toulouse, France; melissa.prat@live.fr (M.P.); marie.salon@wanadoo.fr (M.S.); alaeddine_mohamad@live.fr (T.A.); bernard.pipy@inserm.fr (B.P.)

RESTORE Research Center, Université de Toulouse, INSERM, CNRS, EFS, UPS, 31100 Toulouse, France

3 GamaMabs Pharma, 31106 Toulouse, France; odubreuil@gamamabs.fr (O.D.); Berengere.jean@pierre-fabre.com (B.J.); fanny.lemee@evotec.com (F.L.); jfprost@gamamabs.fr (J.-F.P.); jmbarret@gamamabs.fr (J.-M.B.)

4 Institut Jules Bordet, Université Libre de Bruxelles, 1000 Brussels, Belgium; gregory.noel@bordet.be

5 Univ Angers, Université de Nantes, CHU Angers, Inserm, CRCINA, SFR ICAT, 49000 Angers, France; laurence.preisser@univ-angers.fr (L.P.); pascale.jeannin@univ-angers.fr (P.J.)

6 Laboratory of Immunomonitoring in Oncology, Gustave Roussy, 94905 Villejuif, France; lydie.cassard@gustaveroussy.fr

* Correspondence: agnes.coste@univ-tlse3.fr; Tel.: +33-534-609-501

+ These authors contributed equally to this work.

$\ddagger$ Recently deceased.

Simple Summary: AMHRII, the anti-Müllerian hormone receptor, is selectively expressed in normal sexual organs in healthy adults but is also re-expressed in ovarian, colorectal and lung cancers. In this context, we developed murlentamab, a humanized glyco-engineered anti-AMHRII monoclonal antibody, currently in clinical trial. Preliminary data suggest that murlentamab anti-tumor activity involves immune response activation. Thus, in vitro experiments were performed to precisely characterize the murlentamab effect on the human immune system. We show that murlentamab treatment is associated with evidences of innate and adaptive immune cell activation in cancer patient samples. Moreover, we demonstrate that the murlentamab opsonization of AMHRII-expressing ovarian tumor cells promotes a polarization switch of both naïve and tumor-associated macrophages towards an anti-tumor M1-like phenotype. Our work also supports that, through macrophage reeducation, murlentamab activates an anti-tumor adaptive immune response. Finally, the combination of murlentamab with pembrolizumab confirmed novel clinical perspectives of murlentamab association with checkpoint inhibitors and other immuno-modulators.

Abstract: AMHRII, the anti-Müllerian hormone receptor, is selectively expressed in normal sexual organs but is also re-expressed in gynecologic cancers. Hence, we developed murlentamab, a humanized glyco-engineered anti-AMHRII monoclonal antibody currently in clinical trial. Lowfucosylated antibodies are known to increase the antibody-dependent cell-mediated cytotoxicity (ADCC) and antibody-dependent cellular phagocytosis (ADCP) potency of effector cells, but some preliminary results suggest a more global murlentamab-dependent activation of the immune system. In this context, we demonstrate here that the murlentamab opsonization of AMHRII-expressing ovarian tumor cells, in the presence of unstimulated- or tumor-associated macrophage (TAM)-like macrophages, significantly promotes macrophage-mediated ADCC and shifts the whole microenvironment towards a pro-inflammatory and anti-tumoral status, thus triggering anti-tumor activity. We also report that murlentamab orients both unstimulated- and TAM-like macrophages to an M1-like phenotype characterized by a strong expression of co-stimulation markers, pro-inflammatory cytokines and chemokines, favoring $\mathrm{T}$ cell recruitment and activation. Moreover, we show that 
murlentamab treatment shifts $\mathrm{CD} 4{ }^{+} \mathrm{Th} 1 / \mathrm{Th} 2$ balance towards a Th1 response and activates $\mathrm{CD} 8^{+} \mathrm{T}$ cells. Altogether, these results suggest that murlentamab, through naïve macrophage orientation and TAM reprogrammation, stimulates the anti-tumor adaptive immune response. Those mechanisms might contribute to the sustained clinical benefit observed in advanced cancer patients treated with murlentamab. Finally, the enhanced murlentamab activity in combination with pembrolizumab opens new therapeutic perspectives.

Keywords: murlentamab; glyco-engineered antibody; tumor-associated macrophages; ovarian cancer; adaptive immunity; immunotherapy

\section{Introduction}

The Anti-Müllerian hormone type II receptor (AMHRII), also known as MIS type II receptor (MISRII or MISIIR), is a member of the transforming growth factor beta (TGF- $\beta$ ) receptor superfamily [1,2]. AMHRII plays a major role in male fetus sexual differentiation by inducing, as a consequence of $\mathrm{AMH}$ stimulation, the regression of Müllerian ducts, precursors of female reproductive organs (uterus, fallopian tubes and upper vagina) [3]. In adults, AMHRII seems to have a restricted expression profile, being mainly expressed in granulosa cells in women from birth to menopause, acting in follicular growth modulation [4] and expressed in Sertoli and Leydig cells of males with involvement in androgen biosynthesis regulation [5]. Several studies have confirmed the expression of AMHRII in gynecological cancer tissues [6-10] and, more recently, AMHRII was found expressed by certain non-gynecological cancer such as non-small cell lung cancer and colorectal cancer [11,12]. This set of consistent evidence led to the development of murlentamab, also named GM102 or 3C23K, a humanized glyco-engineered anti-AMHRII monoclonal antibody. Following an extensive pharmacological profiling as well as toxicological studies in cynomolgus monkeys [12-14], a phase I study of murlentamab in gynecological cancers (www.clinicaltrial.gov / NCT02978755, accessed on: 20 June 2016) and a phase IIa in colorectal cancer (www.clinicaltrial.gov/NCT03799731, accessed on: 11 July 2018) are currently ongoing.

Effector functions mediated by the Fc part of immunoglobulins have been reported to be strongly related to their $N$-linked oligosaccharide structures [15]. Particularly core fucosylated oligosaccharides showed weaker binding to the Fc $\gamma$ RIIIa receptor (CD16) expressed on effector cells and resulted in a decreased lytic potential [16,17]. This important therapeutic notion has led to the development of Fc glyco-engineered antibodies, especially low-fucosylated antibodies, for increasing CD16 affinity, for improving antibodydependent cell-mediated cytotoxicity (ADCC) and antibody-dependent cellular phagocytosis (ADCP) and for compensating the inhibitory effect of the high amount of multi-specific immunoglobulins G (IgGs) from serum of patients [18]. Based on this technology, the anti-CD20 antibody obinutuzumab was the first glyco-engineered antibody approved for chronic lymphocytic leukemia and follicular lymphoma treatment [19,20]. Regarding solid tumors, imgatuzumab, one of the most advanced glyco-engineered antibody, showed promising results in epidermal growth factor receptor (EGFR)-positive solid tumors [21], in head and neck squamous cell carcinoma and in colorectal cancer showing complete and partial response in Phase I and II studies [22,23], especially with tumors highly infiltrated by immune cells at baseline. Interestingly, clinical responses were associated with a robust decrease in peripheral Natural Killer (NK) counts attributed to the migration of NK within the tumor [21-23]. This decrease is the main modulation of the immune system reported with glyco-engineered antibodies in clinic up to now.

Although NK cells are the main effector type in both physiological and therapeutic settings, other myeloid types such as dendritic cells (DC), neutrophils and monocytes/macrophages are also involved in ADCC [24]. Because of their noticeable plasticity which allows them to perform several functions within the tumor microenvironment, 
macrophages represent promising therapeutic targets for cancer treatment. Indeed, accumulating preclinical and clinical observations demonstrated that modulating macrophage polarization in the tumor microenvironment may represent an additional approach for cancer treatment, either alone or in combination with immune checkpoint therapies $[25,26]$. Macrophages tailor their polarization states and responses depending on stimulatory signals received from the tumor stroma [27-31]. Although it is now clearly recognized that they can adopt a wide spectrum of polarization states [32-34], macrophages are generally defined as two extremes: classically activated M1 and alternatively activated M2 cells [35]. M1 macrophages are characterized by the expression of Toll-like receptors (TLRs) and opsonic receptors (e.g., TLR2/4, CD16, CD32, CD64), the production of pro-inflammatory mediators and exhibit a strong anti-tumor activity. Conversely, M2 macrophages express an abundant level of non-opsonic receptors (e.g., CD163, CD36, CD206), produce antiinflammatory cytokines and contribute in many ways to tumor progression [36]. Higher tumor infiltration of these M2 macrophages, also called tumor-associated macrophages (TAMs), is associated with more aggressive tumor characteristics. Although the precise origin of TAMs is still under debate, circulating monocytes represents well-described TAM precursors, revealing monocyte subsets as critical in the acquisition of pro- or anti-tumor functions of TAMs [37]. Recently, Prat and colleagues showed in patients with ovarian cancer that murlentamab treatment is associated with changes in the proportions of blood monocyte subsets [38]. Furthermore, Bougherara et al. assessed the effect of murlentamab on immune cells and suggested that TAMs could be involved in the anti-tumor potential of this antibody in ovarian carcinoma [14]. In this context, understanding the fine-tuned mechanisms by which murlentamab exerts its anti-tumor activity and investigating its impact on macrophage polarization might provide more arguments in favor of its use in cancer therapy.

In this study we show that murlentamab treatment is associated with several evidences of innate and adaptive immune cell activation in cancer patient samples. Moreover, through an in vitro co-culture system, we demonstrate here that the murlentamab opsonization of AMHRII-expressing ovarian tumor cell lines promotes a shift in polarization of both naïve (M0) and TAM-like macrophages towards an anti-tumor M1-like phenotype. Our work also supports that, through macrophage orientation, murlentamab activates an anti-tumor adaptive immune response. Finally, both in vitro and in vivo studies with murlentamab in combination with pembrolizumab confirmed novel clinical perspectives of murlentamab association with checkpoint inhibitors and other immuno-modulators.

\section{Results}

2.1. Murlentamab Treatment of Colorectal and Ovarian Cancer Patients Is Associated with the Activation of the Innate and Adaptive Immune Response

Phase I (C101) then phase IIa (C201) studies were performed with murlentamab to determine recommended doses and treatment responses, characterized by overall survival (OS) and progression-free survival (PFS) (Figure S1). Moreover, these studies allowed to assess pharmacodynamic (PD) markers in patient blood and biopsies that contribute to mechanistically specify the mode of action of murlentamab in human. Thus, patient samples from these two studies were collected, processed and analyzed to determine their cytokinic blood profile by ELISA and the activation of immune cells in both blood and the tumor microenvironment by flow cytometry and immunohistochemistry at baseline and at different time points following murlentamab infusion.

All blood samples of colorectal cancer patients from the Belgian hospital $(n=20)$ were analyzed before (baseline) and during cycle 1 (at D15) and cycle 2 (at D43) of murlentamab treatment. Murlentamab infusion induced both an increase in the proportion of blood monocytes positives for the CD69, an early activation marker, and a decrease in CD69 ${ }^{+}$ activated-regulatory $\mathrm{T}$ cells (Tregs), whilst no significant variation was noticed regarding $\mathrm{CD} 9^{+} \mathrm{CD}^{+} \mathrm{T}$ cells at these times of blood sampling (Figure 1A). Blood samples from ovarian cancer patients were not centralized but tested in each clinical site, using sitespecific markers. Therefore, analysis of blood samples from patients treated at Gustave 
Roussy (Paris, France) $(n=4)$ also showed signs of immune activation characterized by an increased proportion of $\mathrm{CD}^{+} \mathrm{T}$ cells expressing the inducible co-stimulatory molecule (ICOS) in all four patients tested during murlentamab treatment (Figure 1B). In addition, treatment of colorectal cancer patients by murlentamab was associated with an increase in CXCL9 and CXCL10 blood levels, two interferon-inducible chemokines described as critical immune modulators and survival predictors in colorectal cancer [39], in 13 patients out of 16 and 9 patients out of 15, respectively (Figure 1C).

We also evaluated immune markers at the tumor site when patients were volunteers for biopsies before treatment (baseline) and in cycle 2 (at D22). Analysis of eight paired biopsies from C201 showed that murlentamab treatment was associated with a decrease in $\mathrm{CD}_{14}{ }^{+}$cells positive for the CD163 receptor (Figure 1D), a marker generally used to identify TAMs in malignant diseases [40]. Due to the size of biopsies, fewer paired biopsies from C201 could be analyzed with other markers and thus an increase in $\mathrm{ICOS}^{+}$cells was noticed in four out of five patients tested (Figure 1D). In terms of effector cells, four paired biopsies from C201 (Figure 1E) and the two paired biopsies from C101 (Figure 1F) showed that murlentamab treatment induced an increase in the proportion or number of both CD16 $6^{+}$ cells (in the two patients tested from the C101 study and four out of five patients from C201) and $\mathrm{CD}_{16}{ }^{+}$cells expressing Granzyme B (in the two patients from the C101 study and in three out of four patients from C201), suggesting a murlentamab-induced activation of macrophages and/or Natural Killer (NK) cells into the tumor microenvironment. Moreover, in colorectal cancer patients' tumor biopsies (C201), murlentamab treatment was associated with increased proportions of CD8 ${ }^{+}$and ICOS co-stimulatory molecule-positive cells in three out of four biopsies tested (Figure 1E).

A

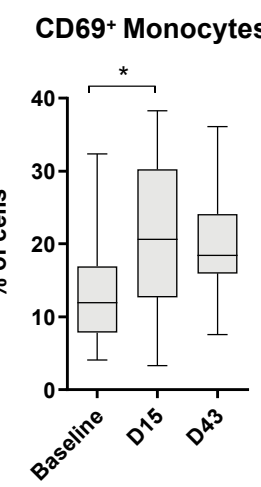

C201

CD69+ regulatory $\mathrm{T}$ cells

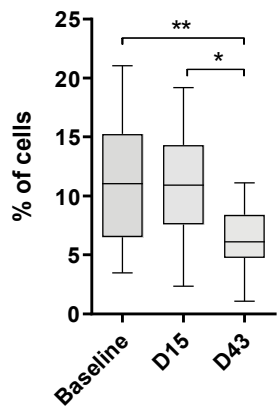

C201

C

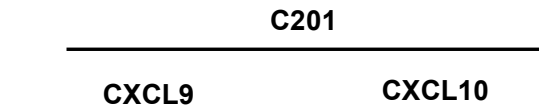

CXCL10

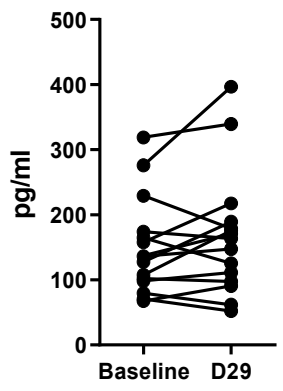

CD69+ $\mathrm{CD} 8+\mathrm{T}$ cells

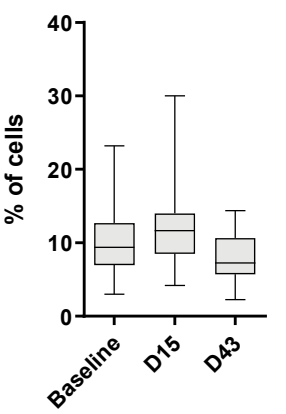

B

C101

$\mathrm{ICOS}^{+} \mathrm{CD}^{+}{ }^{+} \mathrm{T}$ cells

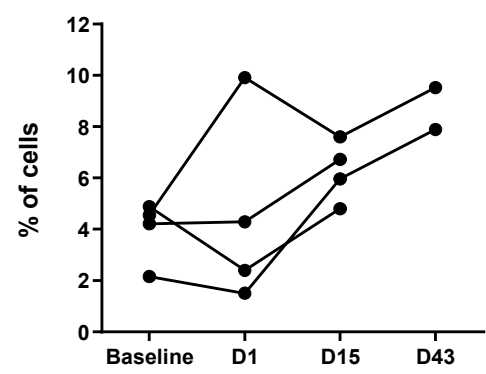

D

C201

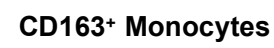

ICOS+ cells
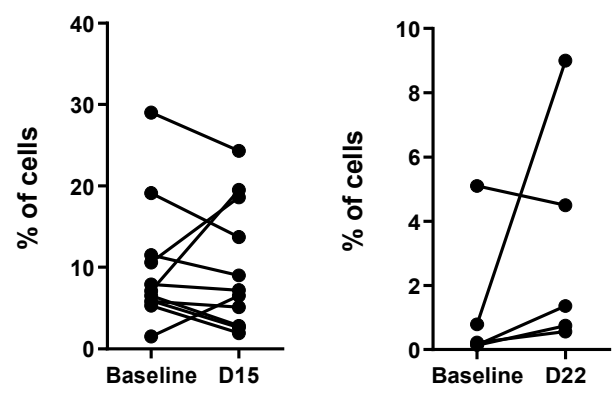

Figure 1. Cont. 
E

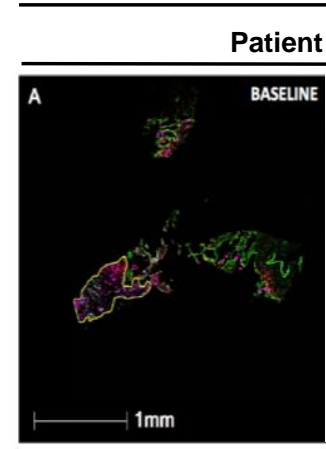

Baseline

Pan-cytokeratin+ - CD16+ - GrZB+ - CD8+

$\mathbf{F}$
C201
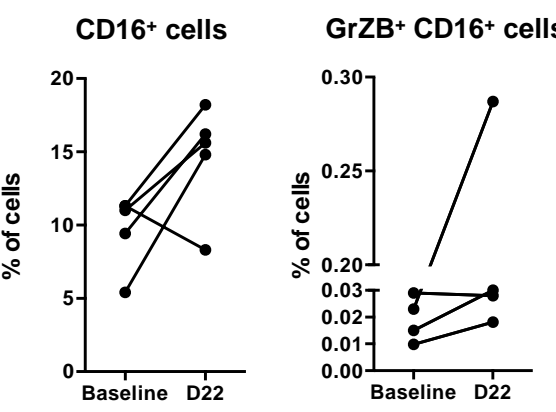

CD8 $+T$ cells

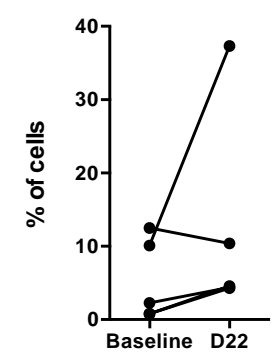

C101
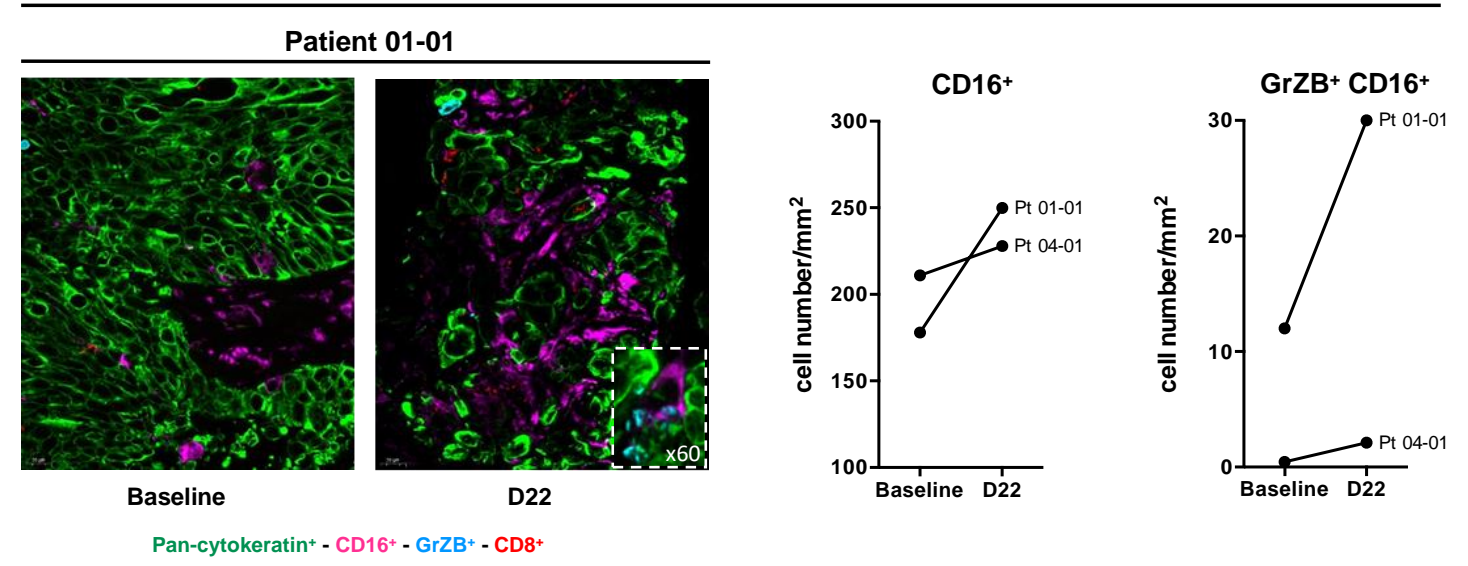

Figure 1. Variation of pharmacodynamic markers measured in blood samples and biopsies of patients from C201 and C101 studies. (A) Detection of CD69 as a marker of activation of monocytes, regulatory and CD8 ${ }^{+} \mathrm{T}$ cells in blood samples of patients $(n=20)$ with colorectal cancer treated with murlentamab (single agent and in combination with trifluridine/tripiracil). Data shown (boxplots) are the results from 20 patients. ${ }^{*} p<0.05$; ${ }^{* *} p<0.01$. $p$ values were determined using one-way ANOVA analysis followed by Tukey's multiple comparisons test. (B) Detection of ICOS (inducible co-stimulatory molecule) as a marker of lymphocyte activation in blood samples of patients with ovarian cancer treated at Gustave Roussy (Paris, France) with murlentamab in combination with carboplatin + paclitaxel $(n=4)$. (C) Detection of CXCL9 $(n=16)$ and CXCL10 $(n=15)$ release in blood samples of all patients treated with murlentamab single agent in C201. (D) Detection of co-staining CD14/CD163 $(n=8)$ and of ICOS $(n=4)$ as markers of immune system regulation in FFPE (Formalin-Fixed Paraffin-Embedded) biopsies obtained from the $\mathrm{C} 201$ study. (E) Image and quantification of CD16 $(n=5)$, co-staining CD16/granzyme B (GrZB) $(n=4)$, and CD8 $(n=4)$ as markers of immune system activation in FFPE biopsies obtained from the C201 study. (F) Image and quantification of CD16/granzyme B (GrZB) co-staining in FFPE biopsies obtained from the C101 study $(n=2)$.

Altogether, these observations from clinical studies of colorectal and ovarian cancer patients strongly suggest that murlentamab treatment induces a local and systemic activation of both innate and adaptive immune cells.

\subsection{Murlentamab Enhances Nä̈ve Macrophage and Tumor-Associated Macrophage Intrinsic Anti-Tumor Activity}

In order to further characterize the precise mechanisms by which murlentamab could impact immune cell activation and given the pivotal role of macrophages within the tumor microenvironment, we first assessed whether murlentamab could promote and/or restore the in vitro anti-tumor activity of naïve macrophages and TAMs.

For this purpose, SKOV3 human ovarian tumor cells modified to strongly express AMHRII (SKOV3-R2 ${ }^{+}$) were opsonized with murlentamab, 3C23K-FcKO (a control anti- 
body mutated in the constant part) or 3C23K-CHO (the 3C23K normal fucose form). These opsonized tumor cells were then co-cultured with naïve unstimulated human monocytederived macrophages (MDMs) (M0) or M-CSF/IL-10-treated human MDMs used to mimic TAMs found in the tumor microenvironment [41,42] (Figure S2).

As illustrated in Figure 2A, irrespective of whether the co-culture was done with unstimulated (M0) or TAM-like MDMs, the number of SKOV3-R2 ${ }^{+}$tumor cells opsonized with the $3 \mathrm{C} 23 \mathrm{~K}-\mathrm{FcKO}$ control increased over time. Interestingly, while the opsonization of SKOV3-R2 ${ }^{+}$tumor cells with the $3 \mathrm{C} 23 \mathrm{~K}-\mathrm{CHO}$ did not modify the kinetic of tumor cell number, we observed a significant reduced number of murlentamab-opsonized SKOV3-R2 ${ }^{+}$ at D4 in the presence of M0 MDMs and at both D4 and D5 in the presence of TAM-like MDMs with respect to $3 \mathrm{C} 23 \mathrm{~K}-\mathrm{FcKO}$ (Figure 2A). These results suggest that the murlentamab opsonization of ovarian tumor cells promotes and/or restores the direct anti-tumor activity of naïve macrophages and TAMs.

ADCC is a well-described function of macrophages and NK cells that has been attributed to the anti-tumor activity of several antibodies used for cancer immunotherapy [24]. In this context, we explored whether the murlentamab opsonization of ovarian tumor cells could trigger human MDM-dependent ADCC. Interestingly, we showed that opsonization of SKOV3-R2 ${ }^{+}$tumor cells with murlentamab strongly triggered ADCC mediated by both unstimulated (M0) and TAM-like macrophages, whereas $3 \mathrm{C} 23 \mathrm{~K}-\mathrm{CHO}$ was only able to slightly induce ADCC mediated by TAM-like macrophages (Figure 2B). These results demonstrate that the opsonization of tumor cells by murlentamab significantly promote and/or restore the ADCC anti-tumor mechanism in both naïve macrophages and TAMs.
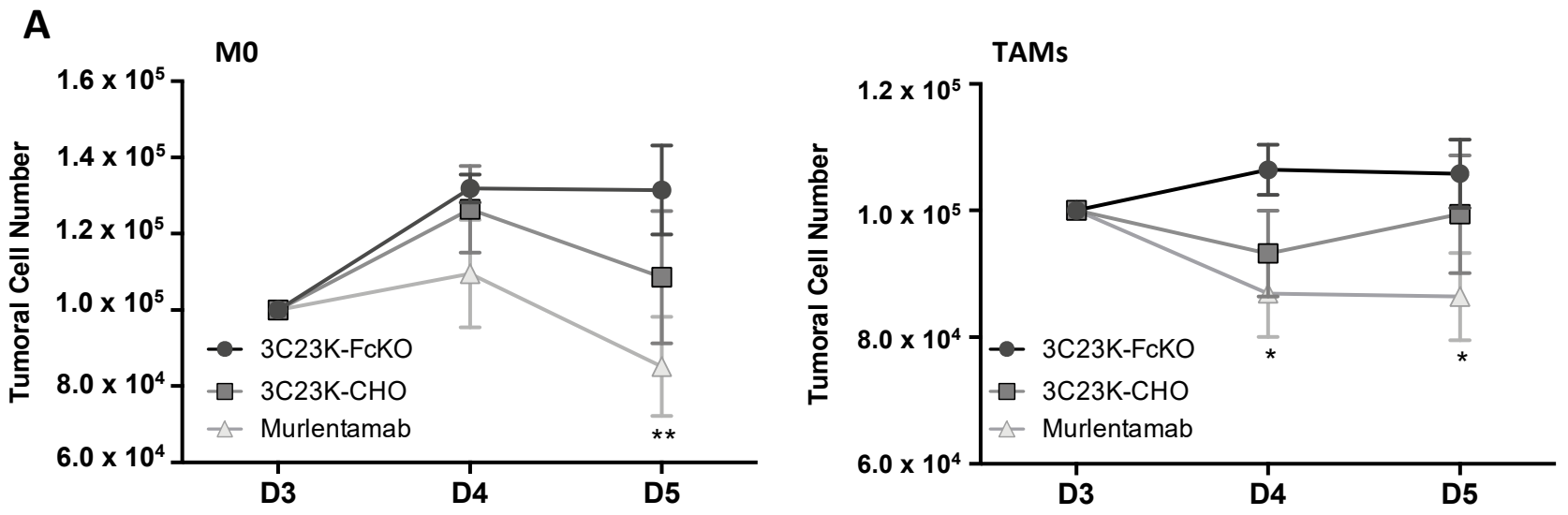

\begin{tabular}{|c|c|c|c|c|c|c|c|c|}
\hline \multicolumn{9}{|c|}{ Tumor cell number M0 } \\
\hline \multicolumn{3}{|c|}{ 3C23K-FcKO } & \multicolumn{3}{|c|}{$3 \mathrm{C} 23 \mathrm{~K}-\mathrm{CHO}$} & \multicolumn{3}{|c|}{ Murlentamab } \\
\hline D3 vs. D4 & * & 0.0158 & D3 vs. D4 & ns & 0.2916 & D3 vs. D4 & ns & 0.2168 \\
\hline D3 vs. D5 & * & 0.0171 & D3 vs. D5 & ns & 0.8686 & D3 vs. D5 & ns & 0.9444 \\
\hline D4 vs. D5 & ns & 0.9991 & D4 vs. D5 & ns & 0.5556 & D4 vs. D5 & ns & 0.3142 \\
\hline \multicolumn{9}{|c|}{ Tumor cell number TAMs } \\
\hline \multicolumn{3}{|c|}{ 3C23K-FcKO } & \multicolumn{3}{|c|}{$3 \mathrm{C} 23 \mathrm{~K}-\mathrm{CHO}$} & \multicolumn{3}{|c|}{ Murlentamab } \\
\hline D3 vs. D4 & * & 0.027 & D3 vs. D4 & ns & 0.7514 & D3 vs. D4 & ns & 0.8514 \\
\hline D3 vs. D5 & * & 0.0179 & D3 vs. D5 & ns & 0.9979 & D3 vs. D5 & ns & 0.8769 \\
\hline D4 vs. D5 & $\mathrm{ns}$ & 0.0646 & D4 vs. D5 & ns & 0.7867 & D4 vs. D5 & ns & 0.9985 \\
\hline
\end{tabular}

Figure 2. Cont. 


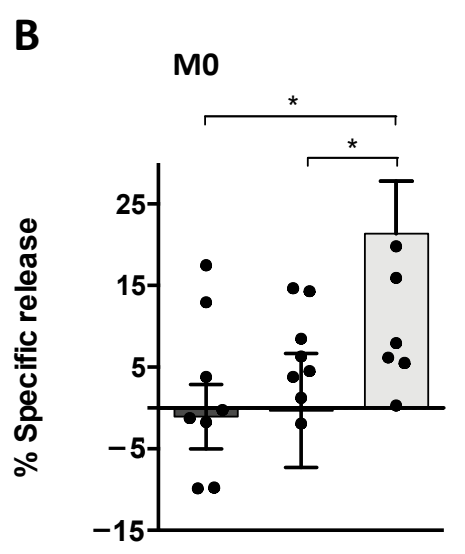

ADCC
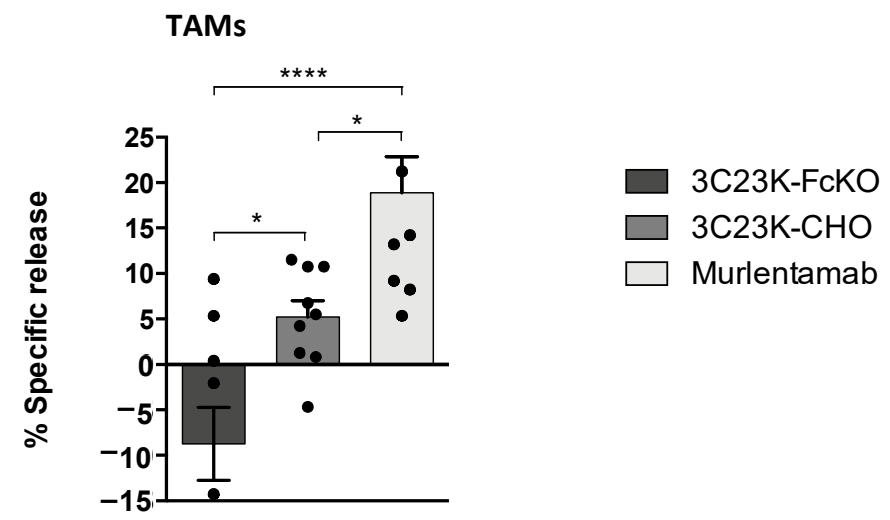

Figure 2. Murlentamab opsonization of SKOV3-R2 ${ }^{+}$increases macrophage anti-tumoral activity and antibody-dependent cell-mediated cytotoxicity (ADCC) killing. SKOV3-R2 ${ }^{+}$ovarian tumor cells were labeled with different 3C23K antibodies (3C23K-FcKO control, 3C23K-CHO normally fucosylated or murlentamab the low fucosylated form) and cultured in the presence of human monocyte-derived macrophages from healthy donors unstimulated (M0) or stimulated with M-CSF and IL-10 (tumor-associated macrophages (TAMs)). (A) Opsonized-SKOV3-R2 ${ }^{+}$cell number was determined by flow cytometry after one and two days of co-culture with M0 or TAMs. ${ }^{*} p<0.05 ;{ }^{* *} p<0.01$ compared 3C23K-FCKO vs. murlentamab at a given time (B) ADCC was performed after $4 \mathrm{~h}$ of co-culture between SKOV3-R2 ${ }^{+}$cells and M0 or TAMs. Data shown (mean $\pm \mathrm{SEM}$ ) are the results from three different experiments (performed with three different healthy donors). ${ }^{*} p<0.05$; **** $p<0.0001$. $p$ values were determined using one-way ANOVA analysis followed by Tukey's multiple comparisons test.

\subsection{Murlentamab Orients Nä̈ve Macrophages and Reprograms Tumor-Associated Macrophages} towards an M1-Like Profile

Following the previous results regarding clinical studies and considering the study of Bougherara et al. implying an activation of T cells through MDMs in the murlentamabinduced killing of tumor cells [14], we further evaluated in vitro whether the murlentamab opsonization of human ovarian tumor cells could impact macrophage phenotype.

After 3 days of co-culture with opsonized SKOV3-R2 ${ }^{+}$tumor cells, the proportion of MDMs expressing M1 or M2 markers was evaluated by flow cytometry (see Table 1 for antibodies used) and their ability to produce pro- and anti-inflammatory mediators was assessed by AlphaLisa immunoassays (Figure S2).

Table 1. Flow cytometry antibodies used.

\begin{tabular}{|c|c|c|c|c|}
\hline Antibodies & Supplier & Catalog No. & Clone No. & Experiment \\
\hline CD4-AF700 & Thermofisher & $56-0049-42$ & RPA-T4 & $\mathrm{CD} 9^{+}$detection \\
\hline CD15-eF450 & Thermofisher & $48-0158-42$ & MMA & \\
\hline CD16-PC7 & Thermofisher & $25-0168-42$ & CB16 & \\
\hline CD25-PerCP & Thermofisher & $46-0257-42$ & CD45-4E3 & \\
\hline CD56-FITC & Thermofisher & $61-0567-42$ & CMSSB & \\
\hline CD64-PerCPeF710 & Thermofisher & $46-0649-42$ & 10.1 & \\
\hline CD69-PE & Thermofisher & $12-0699-42$ & FN50 & \\
\hline CD127-APC & Thermofisher & $17-1278-42$ & eBioRD5 & \\
\hline CD3-VB & Miltenyi & 130-133-133 & BW264/56 & \\
\hline CD45-VG & Miltenyi & $130-113-124$ & $5 \mathrm{~B} 1$ & \\
\hline CD14- AF714 & Miltenyi & 130-113-144 & TUK4 & \\
\hline CD8-PEeF610 & BD BioSciences & 563919 & SK1 & \\
\hline CD3-AA700 & Beckman & B10823 & UCHT1 & $\mathrm{ICOS}^{+}$detection \\
\hline CD8-KrO & Beckman & B00067 & B9.11 & \\
\hline CD4-PB & Beckman & A99020 & RMO52 & \\
\hline HLA-DR-ECD & Beckman & A66330 & $3 \mathrm{G} 8$ & \\
\hline
\end{tabular}


Table 1. Cont.

\begin{tabular}{|c|c|c|c|c|}
\hline Antibodies & Supplier & Catalog No. & Clone No. & Experiment \\
\hline CD45RA-PE & AbD Serotec & MCA1075F & AT10 & \\
\hline ICOS-APC & Beckman & IM1239U & $84 \mathrm{H} 10$ & \\
\hline PD1 & Cell Signaling & $13684 S$ & EIL3N & In vivo experiment \\
\hline CD45-BV421 & BD BioSciences & 563879 & HI30 & \\
\hline CD3-FITC & Miltenyi & $130-098-162$ & REA613 & \\
\hline CD14-PE & Miltenyi & $130-100-676$ & REA599 & \\
\hline CD163-APC & Miltenyi & $130-112-129$ & GHI:61.1 & \\
\hline CD86-Alexa700 & BD BioSciences & 561124 & 2331 & \\
\hline CD11b-APCVio770 & Miltenyi & $130-110-556$ & REA713 & \\
\hline $\begin{array}{c}\text { Viobility }^{\mathrm{TM}} \text { Fixable } \\
\text { Dyes }\end{array}$ & Miltenyi & $130-109-816$ & - & In vitro experiments \\
\hline CD11b-FITC & Miltenyi & $130-110-552$ & REA713 & \\
\hline CD163-PE & Miltenyi & $130-112-286$ & REA812 & \\
\hline CD36-PEVio770 & Miltenyi & $130-110-742$ & REA770 & \\
\hline CD206-APC & Miltenyi & 130-124-012 & DCN 228 & \\
\hline CD64-PerCPVio770 & Miltenyi & $130-116-303$ & REA978 & \\
\hline CD80-PE & Miltenyi & $130-123-253$ & REA661 & \\
\hline CD32-PEVio770 & Miltenyi & 130-097-506 & 2E1 & \\
\hline CD282-APC & Miltenyi & $130-120-138$ & REA109 & \\
\hline CD14-APCVio770 & Miltenyi & $130-110-552$ & REA599 & \\
\hline CD183-APC & Miltenyi & $130-120-450$ & REA232 & \\
\hline CD3-APCVio770 & Miltenyi & $130-113-136$ & REA613 & \\
\hline CD4-VioBright FITC & Miltenyi & $130-113-791$ & REA623 & \\
\hline CD25-PE & Miltenyi & $130-115-534$ & REA945 & \\
\hline CD8-PEVio770 & Miltenyi & $130-110-680$ & REA734 & \\
\hline CD45-VioGreen & Miltenyi & $130-110-638$ & REA747 & \\
\hline
\end{tabular}

Regarding Fc-gamma receptor M1 polarization markers, almost all unstimulated (M0) and TAM-like MDMs expressed the CD32 receptor whatever the culture conditions (Figure 3A). Hence, the murlentamab treatment did not significantly impact this high proportion of CD32+ $\mathrm{M} 0$ and TAM-like MDMs (Figure 3A). However, SKOV3-R2 ${ }^{+}$ opsonization with $3 \mathrm{C} 23 \mathrm{~K}-\mathrm{CHO}$ and murlentamab increased the proportion of TAMs expressing the CD64 receptor as compared with the opsonization with the $3 \mathrm{C} 23 \mathrm{~K}-\mathrm{FcKO}$ (Figure 3A). Moreover, the co-culture with SKOV3-R2 ${ }^{+}$cells opsonized with $3 \mathrm{C} 23 \mathrm{~K}-\mathrm{CHO}$ and murlentamab also resulted in a strong induction of the proportion of cells expressing the CD80 co-stimulation molecules and the TLR2 receptor in both M0 and TAMs (Figure 3A). Mirroring this increase in cells expressing M1 markers, the co-culture with SKOV3-R2 $2^{+}$cells opsonized with $3 \mathrm{C} 23 \mathrm{~K}-\mathrm{CHO}$ and murlentamab decreased the proportion of M0 MDMs expressing CD163, CD36 and CD206, three M2 markers involved in TAM tumor promoting and immunosuppressive functions. Interestingly, regarding these M2 markers, a better efficiency of murlentamab compared to the normal fucosylated form $3 \mathrm{C} 23 \mathrm{~K}-\mathrm{CHO}$ was demonstrated (Figure 3A). Moreover, although the opsonization of tumor cells with murlentamab did not modify the proportion of TAMs expressing CD163, it decreased the proportion of TAMs positive for CD36 and CD206 (Figure 3A).

Consistently with the increased proportion of MDMs expressing M1 markers, we observed that the co-culture of murlentamab-opsonized SKOV3-R2 ${ }^{+}$tumor cells with both M0 and TAM-like MDMs induced a significant increase in IL1 $\beta$, IL6 and IFN $\gamma$ pro-inflammatory cytokine production (Figure 3B). Moreover, IL12 was only induced by the the co-culture of murlentamab-opsonized SKOV3-R2 ${ }^{+}$tumor cells with $\mathrm{M} 0$ while TNF $\alpha$ secretion was not modified (Figure 3B). Inversely, the production of the IL10 antiinflammatory cytokine was significantly reduced by the co-culture of M0 MDMs with SKOV3-R2 ${ }^{+}$cells opsonized with murlentamab and $3 \mathrm{C} 23 \mathrm{~K}-\mathrm{CHO}$ with a better efficiency 
of the murlentamab low-fucosylated form, whereas no effects were demonstrated on TAM-like MDMs (Figure 3B).

Regarding pro-inflammatory chemokines involved in the attraction of innate and adaptive immune cells, the production of CCL2 was increased by the co-culture of TAMlike MDMs with SKOV3-R2 ${ }^{+}$cells opsonized with murlentamab (Figure 3B). Moreover, we observed that the production of CCL4, CCL5, CXCL9 and CXCL10 chemokines was induced by the co-culture of murlentamab-opsonized SKOV3-R2 ${ }^{+}$tumor cells with both M0 and TAM-like MDMs, while the normal fucosylated form 3C23K-CHO did not demonstrate any effects (Figure 3B).

In order to unequivocally establish that changes in secreted cytokines observed in the culture medium were linked to a modification of the secretory profile of macrophages rather than tumor cells, TAMs were cultured without any tumor cells in the presence of immobilized antibodies (Figure S3). In line with previous results, the culture of TAMs with the murlentamab antibody induced a significant increase in TNF $\alpha$ and IL6 pro-inflammatory cytokines as well as a decrease in IL10 anti-inflammatory cytokine production (Figure S3).

Altogether, these data demonstrate that the murlentamab opsonization of SKOV3-R2 ${ }^{+}$ ovarian tumor cells is able to orient naïve macrophages and, even more, to reprogram the phenotype of TAMs towards an M1-like profile. Moreover, this reprogrammation seems more effective in the presence of the low-fucose murlentamab compared to its normal fucosylated form (3C23K-CHO). Interestingly, these results showing a strong increased production of several immunostimulatory mediators (i.e., IL-12, IFN IL6, IFN $\gamma$, CXCL9 and CXCL10), also suggest that murlentamab could promote the ability of macrophages to recruit and/or activate the adaptive immune response.

A

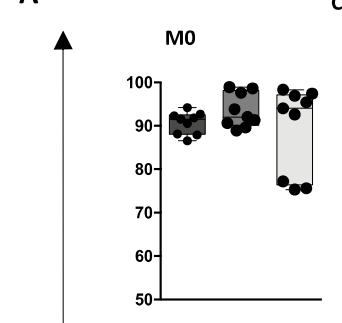

CD32

TAMs

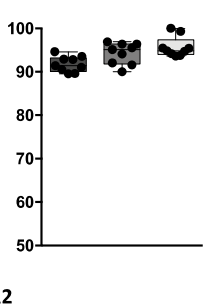

:
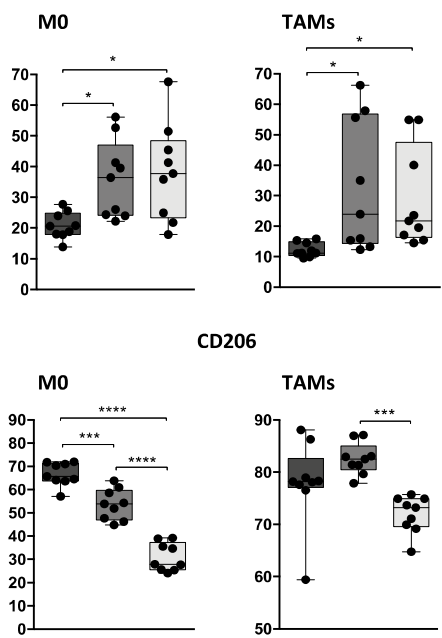

CD206

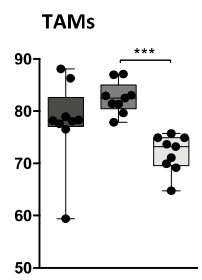

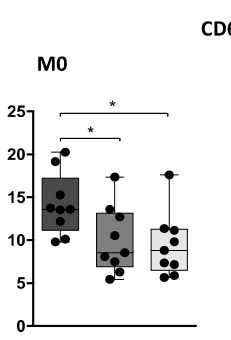

CD64

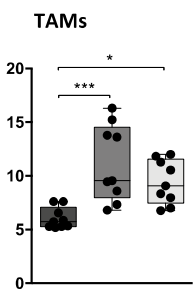

CD163

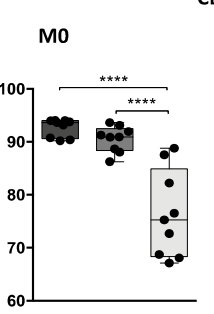

TAMs

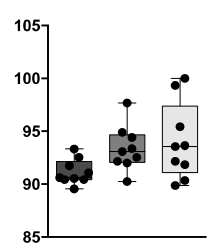

CD80
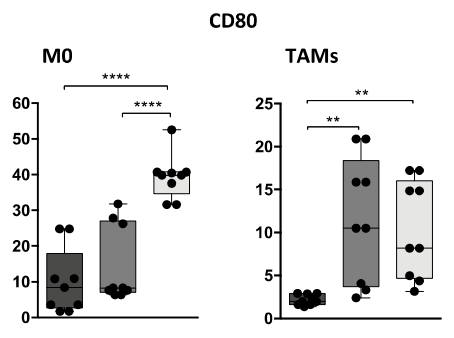

CD36

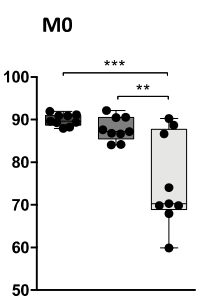

TAMs

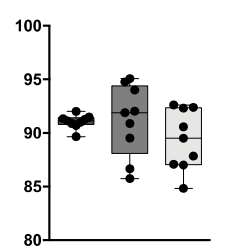

$3 \mathrm{C} 23 \mathrm{~K}-\mathrm{FcKO}$

3C23K-CHO

$\square$ Murlentamab

Figure 3. Cont. 


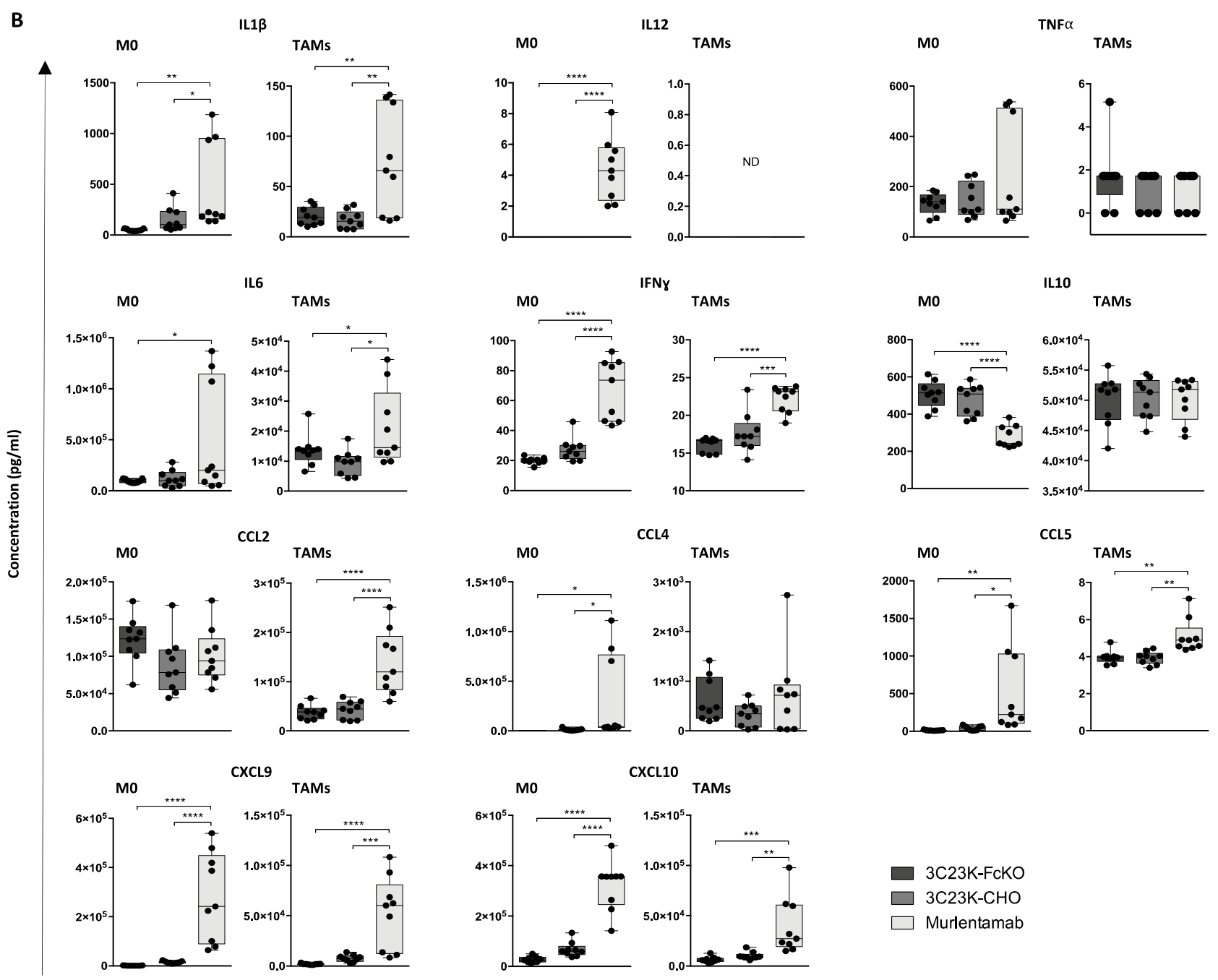

Figure 3. Murlentamab opsonization of SKOV3-R2 ${ }^{+}$orients naïve macrophages and reprograms TAMs towards an M1-like profile. SKOV3-R2 ${ }^{+}$ovarian tumor cells were labeled with different $3 \mathrm{C} 23 \mathrm{~K}$ antibodies $(3 \mathrm{C} 23 \mathrm{~K}-\mathrm{FcKO}$ control, 3C23K-CHO normally fucosylated or murlentamab the low fucosylated form) and cultured in the presence of human monocyte-derived macrophages from healthy donors unstimulated (M0) or stimulated with M-CSF and IL-10 (TAMs). (A) The proportion of macrophages expressing M1/M2 membrane markers (CD32, CD64, CD80, TLR2, CD163, CD36 and CD206) was determined by flow cytometry after three days of co-culture with SKOV3-R2 ${ }^{+}$cells. (B) The release of cytokines (IL1 $\beta$, IL12, TNF $\alpha$, IL6, IFN $\gamma$, IL10) and chemokines (CCL2, CCL4, CCL5, CXCL9 and CXCL10) in the culture medium was determined by AlphaLISA after three days of co-culture with SKOV3-R2 ${ }^{+}$cells. Data shown (boxplots) are the results from three different experiments (performed with three different healthy donors). ${ }^{*} p<0.05 ;{ }^{* *} p<0.01$; ${ }^{* * *} p<0.001 ;{ }^{* * * *} p<0.0001 . p$ values were determined using one-way ANOVA analysis followed by Tukey's multiple comparisons test.

\subsection{Murlentamab Promotes the Ability of Naïve and Tumor-Associated Macrophages to Activate} the Anti-Tumor Adaptive Immune Response

Regarding our results showing that the murlentamab opsonization of SKOV3-R2 ${ }^{+}$ ovarian tumor cells promote the orientation of macrophages towards an M1-like profile expressing soluble mediators involved in T cell recruitment and/or activation (Figure 3), we explored its impact on the adaptive immune cells, especially $\mathrm{CD} 4^{+}, \mathrm{CD} 8^{+}$and Tregs. For this purpose, autologous human $\mathrm{T}$ cells were added in the co-culture with human unstimulated or TAM-like MDMs and SKOV3-R2 ${ }^{+}$tumor cells opsonized with the different antibodies previously described (murlentamab, 3C23K-FcKO control or 3C23K-CHO). At day 10, after 4 days of co-culture, the phenotypic profile of $\mathrm{CD} 4^{+}$, the activation of $\mathrm{CD} 8^{+}$, as well as the proportion of Tregs were evaluated using flow cytometry (Figure S2). 
As a first step, we investigated whether the co-culture with $\mathrm{T}$ cells altered the ability of human MDMs to release pro-inflammatory cytokines and chemokines (Figure S4). While the addition of $\mathrm{T}$ cells into co-culture promoted the ability of TAM-like MDMs cultivated with murlentamab-opsonized SKOV3-R2 ${ }^{+}$to release IFN $\gamma$, IL6 and IL23, it decreased their ability to produce IL10 (Figure S4). Moreover, IL12 and IFN $\gamma$ secretions were induced in M0 MDMs cultivated with murlentamab-opsonized SKOV3-R2 ${ }^{+}$. We also observed that the addition of T cells into co-culture induced the ability of both M0 and TAM-like MDMs cultivated with murlentamab-opsonized SKOV3-R2 ${ }^{+}$to release high amounts of CCL4, CCL5, CXCL9 and CXCL10 pro-inflammatory mediators (Figure S4). This cytokinic signature in favor of a strong immunostimulatory microenvironment suggests a possible murlentamab-dependent increase in the effector T cells (Th1 and Th17) versus Tregs.

Regarding the Th1/Th2 profile of $\mathrm{CD}^{+} \mathrm{T}$ cells, the co-culture of TAM-like MDMs with SKOV3- $2^{+}$cells opsonized with murlentamab and $3 \mathrm{C} 23 \mathrm{~K}-\mathrm{CHO}$ enhanced the proportion of Th1 CD4 ${ }^{+} \mathrm{T}$ cells while lowering the proportion of Th2 $\mathrm{CD}^{+} \mathrm{T}$ cells (Figure $4 \mathrm{~A}$ ). Moreover, the same tendency was observed when murlentamab- and 3C23K-CHO-opsonized SKOV3-R2 ${ }^{+}$tumor cells were co-cultured with M0 MDMs (Figure 4A). Remarkably, we demonstrated that the co-culture of both $\mathrm{M} 0$ and TAM-like MDMs with SKOV-R2 ${ }^{+}$cells opsonized with murlentamab and $3 \mathrm{C} 23 \mathrm{~K}-\mathrm{CHO}$ resulted in a strong reduction of the proportion of Tregs (Figure 4B). Furthermore, in the presence of M0 and TAM-like MDMs, SKOV3-R2 ${ }^{+}$cells opsonized with $3 \mathrm{C} 23 \mathrm{~K}-\mathrm{CHO}$ tended to increase the proportion of CD8 ${ }^{+}$ $\mathrm{CD} 183^{+} \mathrm{T}$ cells, and the opsonization of tumor cells with murlentamab significantly increased activated $\mathrm{CD} 8^{+} \mathrm{T}$ cell proportion (Figure $4 \mathrm{C}$ ).

A Mo
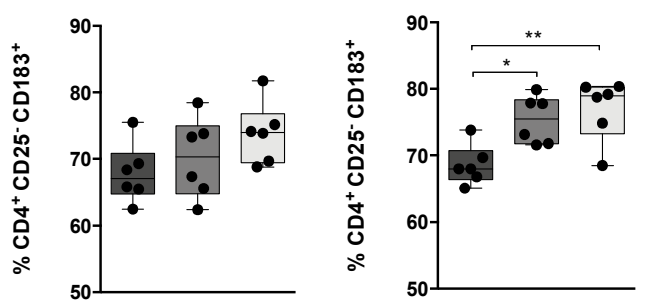

B

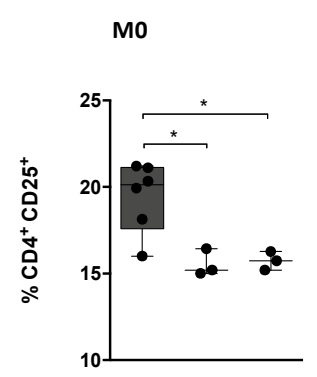

Th1 $\mathrm{CD}^{+} \mathrm{T}$ cells

TAMs

Regulatory $\mathrm{CD}^{+} \mathrm{T}$ cells

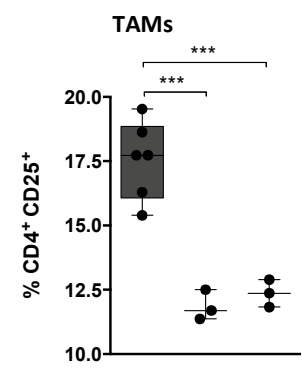

Th2 $\mathrm{CD}^{+} \mathrm{T}$ cells

Mo
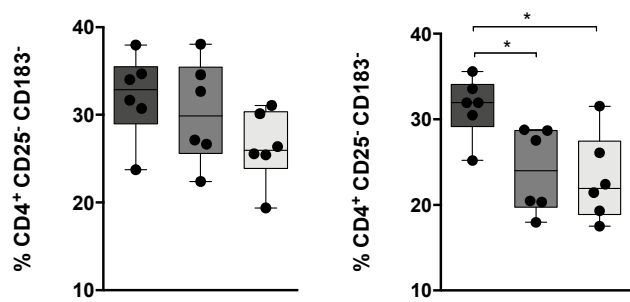

3C23K-FcKO

$3 \mathrm{C} 23 \mathrm{~K}-\mathrm{CHO}$

C

$\mathrm{CD}^{+} \mathrm{T}$ cell activation

Murlentamab

Figure 4. Murlentamab opsonization of SKOV3-R2 ${ }^{+}$activates an effective anti-tumor $\mathrm{T}$ cell immune response. SKOV3-R2 ${ }^{+}$ ovarian tumor cells were labeled with different 3C23K antibodies (3C23K-FcKO control, 3C23K-CHO normally fucosylated or murlentamab the low fucosylated form) and cultured in the presence of human monocyte-derived macrophages from healthy donors unstimulated (M0) or stimulated with M-CSF and IL-10 (TAMs). After 3 days of co-culture, activated T cells coming from the same healthy donor were added in the culture well for 4 more days. (A) The CD4 ${ }^{+}$Th1/Th2 polarization profile, $(\mathbf{B})$ the proportion of $\mathrm{CD} 3^{+} \mathrm{CD} 4^{+} \mathrm{CD} 25^{+}$regulatory $\mathrm{T}$ cells and $(\mathbf{C})$ the activation of $\mathrm{T} \mathrm{CD} 8^{+}$cells were determined by flow cytometry after four days of co-culture. Data shown (boxplots) are the results from two different experiments (performed with two different healthy donors). ${ }^{*} p<0.05 ;{ }^{* *} p<0.01 ;{ }^{* * *} p<0.001$. $p$ values were determined using one-way ANOVA analysis followed by Tukey's multiple comparisons test. 
Interestingly, these findings were extended to another human ovarian tumor cell line modified to overexpress AMHRII (COV434-R2 ${ }^{+}$) (Figure S5). Indeed, as observed with SKOV3-R2 ${ }^{+}$cells, we showed an orientation of $\mathrm{CD}^{+}{ }^{+} \mathrm{T}$ cells towards a Th1 profile as well as an activation of $\mathrm{CD}^{+} \mathrm{T}$ cells when murlentamab-opsonized COV434-R2 ${ }^{+}$were cultured with TAM-like MDMs (Figure S5). Altogether, these data indicate that the opsonization of ovarian tumor cells with murlentamab promotes the activation of an effective anti-tumor $\mathrm{T}$ cell immune response.

\subsection{Murlentamab and Pembrolizumab Association Enhances the Anti-Tumor Potential of Murlentamab Monotherapy}

Given the increasing success of immunotherapies, in particular anti-PD-1/PD-L1 antibodies, we assessed both in vitro and in vivo the impact of the association between murlentamab and pembrolizumab, an anti PD-1 antibody, on tumor cell elimination and immune cell activation.

The addition of pembrolizumab in the co-culture between TAM-like MDMs and SKOV3-R2 ${ }^{+}$cells did not change the kinetic of tumor cell number observed with 3C23K-FcKO-opsonized SKOV3-R2 ${ }^{+}$(Figure 5A). Interestingly, while the number of murlentamab-opsonized SKOV3-R2 ${ }^{+}$cells was significantly reduced after 2 days of coculture with TAM-like MDMs as compared to the 3C23K-FcKO-opsonized SKOV3-R2+ cells, the addition of pembrolizumab into the culture medium enhanced the elimination of murlentamab-opsonized SKOV3-R2 ${ }^{+}$cells by decreasing their number from the first day of co-culture (Figure 5A).

As already observed in Figure 4, the opsonization of ovarian tumor cells with murlentamab, in the presence of TAM-like MDMs, promoted the activation of both $\mathrm{CD} 4^{+} \mathrm{T}$ cells towards a Th1 profile and $\mathrm{CD}^{+} \mathrm{T}$ cells (Figure 5B,C). Moreover, the addition of pembrolizumab to 3C23K-FcKO-opsonized SKOV3-R2 ${ }^{+}$similarly promoted Th1 orientation of $\mathrm{CD}^{+} \mathrm{T}$ cells and $\mathrm{CD}^{+} \mathrm{T}$ cell activation. Interestingly, the stimulation of this antitumor adaptive immune response was improved by the addition of pembrolizumab to the murlentamab-opsonized SKOV3-R2 ${ }^{+}$cells co-cultured with TAM-like MDMs (Figure 5B,C), demonstrating a potentiated effect during murlentamab/pembrolizumab combo-therapy.

A

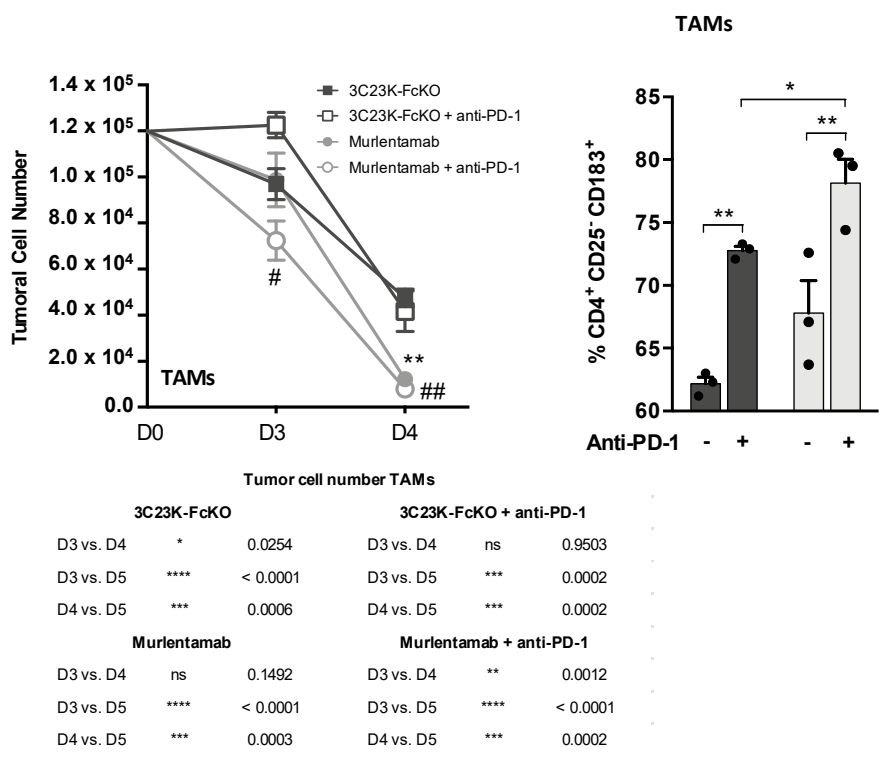

B

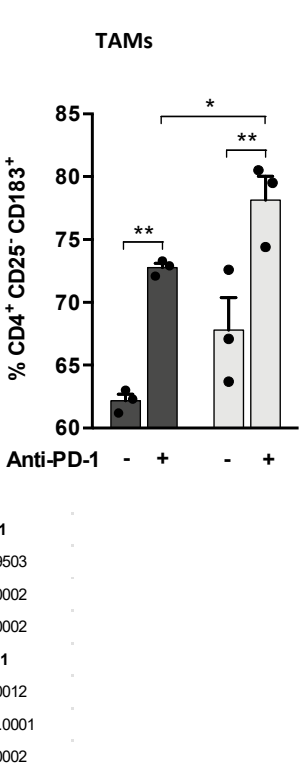

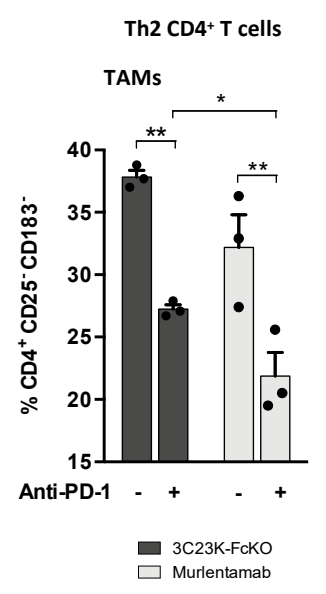

C $\quad$ CD8 ${ }^{+} \mathrm{T}$ cell activation TAMs

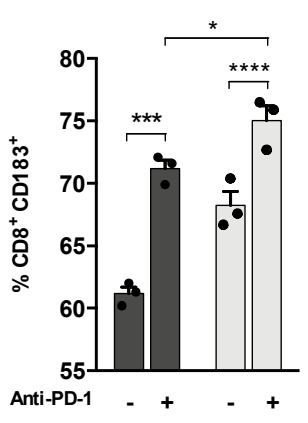

Figure 5. Cont. 
D

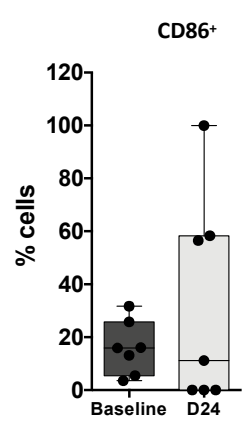

Murlentamab

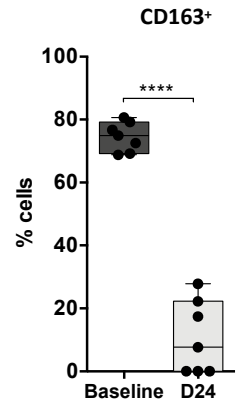

Pembrolizumab

$\mathrm{CD}^{+} 6^{+}$

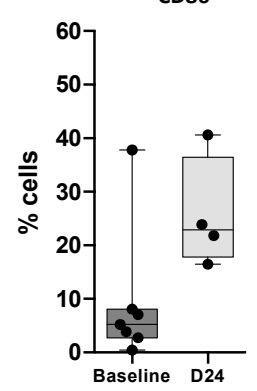

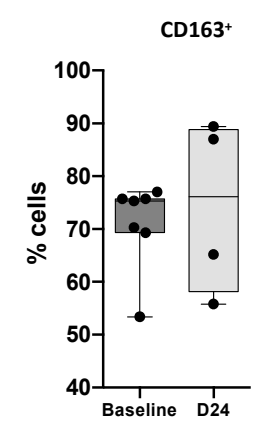

Murlentamab + Pembrolizumab

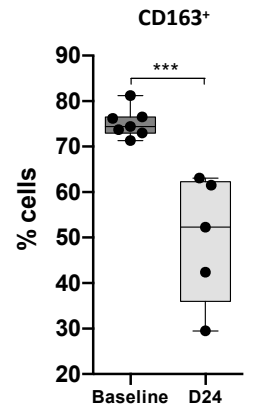

E

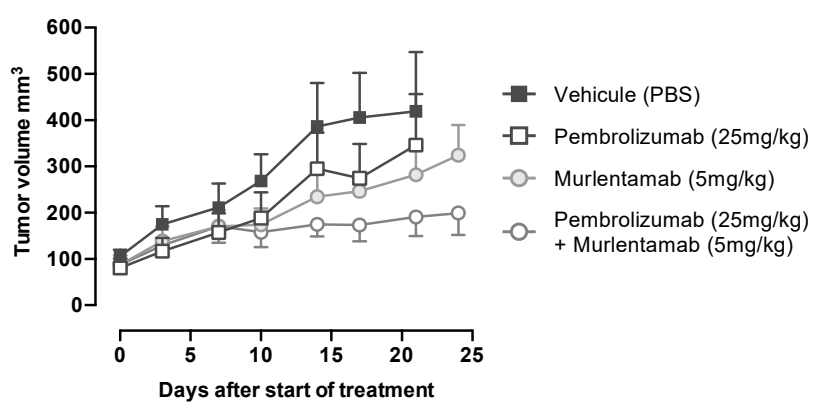

Figure 5. Murlentamab/pembrolizumab combination accentuates the anti-tumoral effect of murlentamab monotherapy through the enhancement of $\mathrm{T}$ cell activation. (A-C) SKOV3-R2 ${ }^{+}$ovarian tumor cells were labeled with different $3 \mathrm{C} 23 \mathrm{~K}$ antibodies (3C23K-FcKO control or murlentamab the low fucosylated form) and cultured in the presence of human monocyte-derived macrophages from healthy donors stimulated with M-CSF and IL-10 (TAMs). After 3 days of co-culture, activated $\mathrm{T}$ cells coming from the same healthy donor were added in the culture well for 4 more days. Pembrolizumab was added into co-culture wells everyday from day 3 to day 10. (A) Opsonized-SKOV3-R2 ${ }^{+}$cell number was determined by flow cytometry after one and two days of co-culture with TAMs. Data shown (mean \pm SEM) are the results from three different experiments (performed with one healthy donors). ${ }^{* *} p<0.01$ compared 3C23K-FcKO vs. Murlentamab. $\# p<0.05$; \#\# $p<0.01$ compared 3C23K-FcKO + anti-PD-1 vs. Murlentamab + anti-PD-1 as determined using one-way ANOVA analysis followed by Dunnett's multiple comparisons test. (B,C) The CD4 ${ }^{+}$Th1/Th2 polarization profile and the activation of $\mathrm{T} \mathrm{CD}^{+}$cells were determined by flow cytometry after four days of co-culture. Data shown (mean $\pm \mathrm{SEM}$ ) are the results from three different experiments (performed with one healthy donors). ${ }^{*} p<0.05 ;{ }^{* *} p<0.01 ;{ }^{* * *} p<0.001$; ${ }^{* * * *} p<0.0001$. $p$ values were determined using one-way ANOVA analysis followed by Tukey's multiple comparisons test. (D,E) $10 \times 10^{6}$ COV434-R2 ${ }^{+}$ovarian tumor cells were transplanted subcutaneously into humanized GM-CSF/IL3/IL4 hu-NOG (NOD/Shi-scid/IL2R $\gamma^{\text {null }}$ ) mice (Taconic). After 35 days, when tumors were big enough, mice were i.p treated or not with murlentamab $(5 \mathrm{mg} / \mathrm{kg})+/$ - pembrolizumab $(25 \mathrm{mg} / \mathrm{kg})$ twice a week for 4 weeks. (D) Quantification of circulating $\mathrm{CD}^{+} 6^{+}$and $\mathrm{CD} 163^{+}$cells by flow cytometry from blood of tumor-bearing mice before treatment and after 24 days of treatment with murlentamab $(5 \mathrm{mg} / \mathrm{kg})$ or pembrolizumab $(25 \mathrm{mg} / \mathrm{kg})$ as single agents or murlentamab/pembrolizumab combo-therapy. Data are represented as boxplots. ${ }^{* *} p<0.001,{ }^{* * * *} p<0.0001$ in comparison to baseline. (E) In vivo tumor growth. Data are represented as mean + SEM.

In order to confirm our in vitro observations, the efficacy of the murlentamab/pembrolizumab combination therapy was then evaluated in vivo using xenografted COV434-R2 ${ }^{+}$ cells, after verification of PD-L1 expression at their surface (data not shown). The assay used was particularly delicate because murlentamab does not cross-react with murine AMHRII. It was therefore necessary to transplant COV434-R2 ${ }^{+}$cells into immunodeficient NOG (NOD/Shi-scid/IL2R $\gamma^{\text {null }}$ ) mice and to re-constitute immune response with an intravenous. injection of human $\mathrm{CD}_{4} 4^{+}$cells. Moreover, to stabilize monocyte population for several weeks in such a model, mice were treated with a GM-CSF/IL3/IL4 combo. This treatment maintained monocytes during all the experiment but, in parallel, favored $\mathrm{GvH}$ reaction. For this reason, the experiment did not exceed 4 weeks and a clear-cut 
anti-tumoral effect related to a murlentamab-dependent immunomodulation was difficult to demonstrate. However, even with these limits, we observed that the treatment of humanized ovarian tumor-bearing mice by murlentamab monotherapy tended to increase $\mathrm{CD} 6^{+}$blood cells and strongly decreased $\mathrm{CD} 163^{+}$blood cells (Figure 5D), signing a monocyte/macrophage orientation towards an M1 anti-tumor profile. Although the pembrolizumab monotherapy did not impact the proportion of blood cells expressing CD86 and CD163, the murlentamab/pembrolizumab association maintained and even more enhanced this M1-like profile (Figure 5D). Consistent with these findings, we observed a tendency towards tumor growth inhibition in mice treated with murlentamab or pembrolizumab monotherapies (Figure 5E). In addition, although not significant (murlentamab vs. combo at D24: $p=0.101$ as determined with the Mann-Whitney one tail test), we showed a trend towards a potentiated effect with the murlentamab/pembrolizumab combo-therapy (Figure 5E). Altogether, these in vitro and in vivo results show a rationale for combining murlentamab with anti-cancer immunomodulators such as pembrolizumab.

\section{Discussion}

Monoclonal antibodies are the fastest growing class of biological therapeutics for the treatment of various cancers. Their strong anti-tumor potential mainly relies on the Fc-mediated immune effector functions, namely, ADCC and ADCP. Reduced core fucosylation of antibodies has been shown to increase IgG1 Fc binding affinity to the CD16a present on immune effector cells (especially NK cells and macrophages) [16], to increase ADCC / ADCP $[17,18]$ and to enhance tumor inhibition in vivo $[43,44]$. These findings led to the production of low-fucose glycol-engineered antibodies for cancer therapy, a family to which murlentamab belongs.

Murlentamab is a humanized low-fucose anti-AMHRII antibody currently under a phase I and a phase IIa clinical evaluation with preliminary data showing anti-tumor activity through a partial response and prolonged PFS [45,46]. In terms of mode of action, preclinical studies of murlentamab demonstrated its high efficiency for binding to the CD16a receptor and for inducing ADCC/ADCP [13,14]. In vivo, this activity was translated into anti-tumor activity against Patient Derived Xenograft (PDX) models and xenografted cells expressing AMHRII $[13,14]$. An ex vivo study has also suggested that murlentamab anti-tumor mechanisms could be also related to the increase in macrophagedependent ADCC/ADCP following CD16a recognition as well as the alleviation of $\mathrm{T}$ cell immunosuppression by these cells [14]. Recently, Prat and colleagues have demonstrated changes in blood monocyte subsets after murlentamab infusion in patients with ovarian cancers [38], thus reinforcing the idea that murlentamab activity could involve monocytes/macrophages.

Following these results, we further investigated the precise in vitro mechanisms involved in this antibody anti-tumor activity with a particular focus on macrophage polarization. Indeed, macrophages can have a dual inhibitory and supportive influence on cancer depending on the disease stage, the tissue involved, the host microbiota and their polarization state whose two extremes are described as M1 and M2 macrophages [47]. Moreover, macrophages play a pivotal role within the tumor microenvironment since, in addition to displaying direct pro- or anti-tumoral activities, they orchestrate the adaptive immune response [47]. In this work, through an in vitro co-culture system allowing the interactions between the different partners involved in the anti-tumor immune response (namely, antibody-opsonized tumor cells, naïve or tumor-educated human MDMs and naïve T cells), we accurately dissected the murlentamab mode of action.

First, we demonstrated that murlentamab treatment resulted in several functional and phenotypic modifications of naïve MDMs (M0). Indeed, as expected and in line with previously cited studies, recognition of murlentamab-opsonized ovarian tumor cells led to a strong ADCC activity of naïve macrophages. Moreover, the murlentamab opsonization of tumor cells induced a phenotypic orientation of M0 macrophages toward an M1-like phenotype. Indeed, in the presence of murlentamab-opsonized tumor cells, macrophages adopt a 
pro-inflammatory profile (secreting IL12, TNF $\alpha$, IL6, IL1 $\beta$, IFN $\gamma$, CXCL9 and CXCL10) with the overexpression of surface receptors characteristic of the M1 polarization (CD80, TLR2) while downregulating several M2-like markers (CD163, CD206, CD36 and IL10). Other studies have already demonstrated that $\mathrm{F} \gamma \mathrm{R}$-mediated signaling has a profound impact on unstimulated monocyte/macrophage. Indeed, engagement of activating Fc $\gamma$ Rs on these cells is associated with the production of pro-inflammatory cytokines and chemokines including IL-8, TNF and IL1 $\beta[48,49]$. In our study, the observed phenotypic switch, although visible with the non-glycoengineered version of the antibody (3C23K-CHO), was potentiated in the presence of the low-fucose antibody (murlentamab). In line with these results showing a glycosylation profile-related effect, Kircheis and colleagues demonstrated that the pro-inflammatory secretion profile of peripheral blood mononuclear cells (PBMCs) was particularly induced using glyco-modified antibody compared to their parental counterpart [49]. Thus, our results confirm that glyco-engineered antibodies not only present a stronger binding to CD16a and a higher lysis potency through ADCC / ADCP but also a greater ability to promote a pro-inflammatory cytokine release.

Moreover, in an original way, we demonstrated here that murlentamab is not only capable of orienting naïve human MDMs towards an M1-like phenotype but also of reprogramming polarized TAMs. Indeed, as previously described with M0 macrophages, the co-culture with murlentamab-opsonized ovarian tumor cells results in (i) an increased TAM ADCC and (ii) a repolarization of TAMs towards an M1 profile secreting a high amount of pro-inflammatory and immunostimulatory cytokines/chemokines. Knowing that tumor cells constantly secrete immunosuppressive factors to educate immune cells [29], these results suggest a promising anti-tumor role of murlentamab in clinical settings, more specifically in cancer patients in whom TAMs represent the major leukocyte infiltrate within a tumor.

In addition to the phenotypic switch of macrophages and the activation of their direct anti-tumor properties, we demonstrated here that murlentamab stimulated an adaptive anti-tumor immunity. Indeed, in co-culture with macrophages and murlentamabopsonized tumor cells, we reported an increased proportion of activated CD8 $8^{+}$and Th1 $\mathrm{CD} 4^{+} \mathrm{T}$ cells as well as a reduced proportion of $\mathrm{CD} 25^{+} \mathrm{CD} 4^{+}$Tregs. This is in line with the pro-inflammatory profile of macrophages which suggest a macrophage-dependent $\mathrm{T}$ cell activation after opsonized tumor cell recognition and $\mathrm{Fc} \gamma \mathrm{R}$ engagement. Indeed, IL12 and IFN $\gamma$ cytokines can affect immune function in several ways, for instance, through (i) the enhancement of cross-presentation by professional antigen-presenting cells, (ii) the augmentation of co-stimulatory molecule expression, including major histocompatibility complex (MHC) I and MHCII, (iii) the polarization of T cells into Th1 effector cell phenotype and (iv) the stimulation of cytotoxic $\mathrm{CD}^{+} \mathrm{T}$ cell proliferation [50,51]. Opening up promising therapeutic perspectives, we also transposed the results obtained in our in vitro model to the clinic. Indeed, through the analysis of two clinical studies carried out in patients with gynecological and advanced/metastatic colorectal cancers, we demonstrated that murlentamab treatment is associated with several signs of innate and adaptive immune cell activation both systemically and locally at the tumor site. Altogether, our results showing an increased CD16/Granzyme B-positive cell infiltration after murlentamab infusion and very few NK cells at the tumor site (in all biopsies tested, less than $0.1 \%$ of cells were positive with a staining using an anti-NKp46 antibody) suggest a monocyte/macrophagedependent anti-tumor mechanism. Supporting this hypothesis, Uchida and colleagues demonstrated that monocytes and/or macrophages and not NK cells are the principal mediators of ADCC against $\alpha$-CD20-coated B cells in vivo [52]. Regarding solid tumors, participation of macrophages in mediating anti-tumor mAb efficacy is less established but at least one report is consistent with this notion [18]. Interestingly, this work further emphasizes the enhanced efficacy of glyco-engineered antibodies (enhanced CD16a binding activity) compared to their wild-type counterpart [18]. Moreover, the decreased proportion of monocytes positive for the CD163, a well-characterized marker of TAMs, supports the murlentamab-dependent modulation of monocytes/macrophage populations/phenotypes. 
Of interest, CD163 expressing TAMs have been linked to poor prognosis, overall survival and metastasis of a range of malignancies, including colorectal and ovarian cancers [39]. Finally, the two clinical studies show some evidence of $\mathrm{T}$ cell activation in patient's blood and tumor biopsies after murlentamab infusion. In this framework, it has already been demonstrated that the mAb-mediated induction of IFN $\gamma$ resulted in DC maturation and increased antigen presentation, which was hypothesized to result in increased cross-presentation potential to $\mathrm{CD} 8^{+} \mathrm{T}$ cells, thereby linking the innate and adaptive immune responses [53]. In addition, the elevation in patients' blood of CXCL9 and CXCL10 chemokines, the levels of which are increased in the co-culture medium after murlentamab-opsonized tumor cell addition, could also partly explain the murlentamab anti-tumor activity by promoting the recruitment of activated $\mathrm{CD} 8^{+}$and $\mathrm{Th} 1$ lymphocytes.

Therapeutic strategies targeting macrophages and aiming to reactivate or re-educate them are currently undergoing clinical assessment [47]. Such strategies have the potential to complement cytoreductive, antiangiogenic, and immune-checkpoint-inhibitor treatments. Interestingly, through in vitro assays and delicate in vivo experiments, our work underscores the improved effect of murlentamab/pembrolizumab bi-therapy compared to murlentamab used as a monotherapy. Moreover, we confirmed in vivo some signs of murlentamab-induced macrophage phenotypic orientation towards an M1-like profile. This polarization shift could contribute to the enhanced effect observed here with pembrolizumab. Although preliminary, these data support the interest of carrying out additional in vivo experiments in order to (i) determine the optimal treatment sequence with these two agents, (ii) compare the activity of murlentamab versus its normal fucosylated form as widely explored in our in vitro studies and (iii) further investigate immune cell activation in both mice blood samples and xenografted tumors. However, this first in vivo experiment already confirmed that murlentamab anti-tumor activity is not only restricted to its ability to promote ADCC/ADCP but also involves a more global activation of the immune system. In this framework, a recently published study highlighted the fundamental role of macrophage-derived CXCR3 ligands for the therapeutic efficacy of immune checkpoint blockade, thus highlighting the potential of manipulating this axis to enhance patient responses [54]. Given our results showing a murlentamab-dependent induction of CXCL9 and CXCL10 macrophage secretion, this stresses the importance of our study and supports the benefits of associating murlentamab with other types of immunotherapies such as antibodies against PD1, PDL1, CTLA4, or CD47.

\section{Materials and Methods}

\subsection{Cell Lines and Reagents}

Cells from the human germ cell tumor cell line COV434 [55] were transfected with the cDNA encoding full-length human AMHRII in the PCMV6 plasmid to stably express AMHRII and to constitute the COV434-R2 ${ }^{+}$cell line, as described by Kersual et al. [8]. Cells were grown in DMEM (Dubecco's Modified Eagle Medium) F12 medium containing $10 \%$ heat-inactivated fetal bovine serum, $0.1 \mathrm{mg} / \mathrm{mL}$ streptomycin, $0.1 \mathrm{IU} / \mathrm{mL}$ penicillin and $0.25 \mu \mathrm{g} / \mathrm{mL}$ amphotericin B. COV434-R2 ${ }^{+}$cells were supplemented with $0.33 \mathrm{mg} / \mathrm{mL}$ geneticin. Cells from the human ovarian adenocarcinoma cell line SKOV3 were also transfected with the cDNA encoding full-length human AMHRII in the pCMV6 plasmid as described with COV434 cells. SKOV3 cells were maintained in DMEM medium supplemented with $10 \%$ heat-inactivated fetal calf serum, $100 \mathrm{U} / \mathrm{mL}$ penicillin and $100 \mu \mathrm{g} / \mathrm{mL}$ streptomycin. All cells were grown at $37^{\circ} \mathrm{C}$ in a humidified atmosphere with $5 \% \mathrm{CO}_{2}$ and the medium was replaced twice a week. Cells were harvested with $0.5 \mathrm{~g} / \mathrm{mL}$ trypsin $/ 0.2 \mathrm{mg} / \mathrm{mL}$ EDTA. All culture media and supplements were purchased from Gibco, Thermo Fisher scientific (Waltham, MA, USA).

\subsection{Patients and Study Design}

The primary analyses derive from two clinical studies: (i) the C101 study, a doseranging phase I study of murlentamab single agent or in combination with carboplatin- 
paclitaxel and its expansion with murlentamab single agent in patients with previously treated advanced or metastatic gynecological cancer (NCT02978755; [45]), and (ii) the C201 study, a phase IIa study of murlentamab single agent or in combination with Lonsurf ${ }^{\circledR}$ (trifluridine/tipiracil) in patients with previously treated advanced or metastatic colorectal cancer (NCT01668784; [46]). In the C101 study (Figure S1A), murlentamab was administered to the patients either every two weeks (q2w): on days 1 (D1) and 15 (D15) of each 4-week cycle, or, as in the C201 study (Figure S1B), every week (q1w): on days 1, 8, 15 and 22 (D1, D8, D15, D22) of each 4-week cycle. Details on study treatment and schedule are provided in Figure S1.

\subsection{Tumor and Blood Specimens}

All human tissues and bloods were obtained according to protocols approved by institutional review boards from each country where the studies where performed (Comite de protections des personnes, Hôpital Tarnier-Cochin, Paris, France; Comité d'éthique, Institut Jules Bordet, Brussels, Belgium; Ethics committee research UZ/KU, Leuven, Belgium; London Chelsea, London, UK; Ethics committee for multicentric clinical trial, Motol hospital, Prague, Czech Republic). For both studies, C101 and C201, biopsies and blood samples were obtained from each patient who provided voluntary written informed consent. Circulating immune cells were analyzed at the first day of the first cycle before murlentamab infusion (baseline, D1) and, at day 15 of cycle 1 (D15) and at day 1 of cycle 3 (D43). In the phase I (C101) study, these cells were analyzed with a panel of specific markers for each investigational site, whilst, in the phase IIa (C201) study, all samples from Belgian investigational sites were centralized at Jules Bordet Institute, Brussels, for analysis. In both studies, few tumor specimens have been collected before murlentamab infusion (baseline) and at the end of cycle 2 (D22). Biopsies were fixed in 10\% formalin solution, processed, and embedded in paraffin.

\subsection{Phenotype Analysis of Blood Circulating Immune Cells of Patients}

In the C201 study, CD69 ${ }^{+}$cells were detected by incubating $100 \mu \mathrm{L}$ of fresh total blood with $20 \mu \mathrm{L}$ of anti-human Fc receptor binding inhibitor (ThermoFisher, Bleiswijk, The Netherlands) for $20 \mathrm{~min}$ on ice and then with fluorescent antibodies for $20 \mathrm{~min}$ at $4{ }^{\circ} \mathrm{C}$ (CD3-VioBlue, CD14-APC-vio770, CD45-VioGreen from Miltenyi Biotec and CD4-Alexa Fluor700, CD15-eF450, CD56-FITC, CD64-PerF710, CD16-PC7, CD25-PercPeF710, CD69-PE, CD127-APC from Thermofisher, Bleiswijk, The Netherlands) according to the manufacturer's suggested dilutions. After incubation, $1 \mathrm{~mL}$ of diluted Red Blood Cell lysis buffer (Miltenyi, Bergisch Gladbach, Germany) was directly added and cells were incubated for $10 \mathrm{~min}$ at room temperature in the dark. After centrifugation, cells were immediately acquired on a GALLIOS 10/3 cytometer (Beckman Coulter, Nyon, Switzerland) and results were analyzed with the Kaluza Flow Cytometry Analysis v1.5 software. CXCL9 and CXCL10 quantification was performed using, respectively, Quantikine ${ }^{\circledR}$ ELISA Human CXCL9/MIG Immunoassay and Quantikine ${ }^{\circledR}$ ELISA Human CXCL10/IP-10 Immunoassay kits (R \& D System, Abingdon, UK) according to manufacturer's protocols.

In the C101 study, the rates of $\mathrm{CD}^{+} \mathrm{T}$ cell activation were determined by ICOS expression according to a similar protocol but using another panel of fluorescent antibodies (CD3-AA700, CD4-PB, CD8-KrO, CD25-PC5.5, CD127-AA750, CD45RA-PC7, CD197-PE, CD95-FITC, CD278-APC, HLA-DR-ECD from Beckman Coulter, Marseille, France). The rates of naïve and memory $\mathrm{T}$ cells, Tregs and $\mathrm{T}$ cell activation were determined by HLA-DR and ICOS expression according to a similar protocol but using another panel of fluorescent antibodies (CD3-AA700, CD4-PB, CD8-KrO, CD25-PC5.5, CD127-AA750, CD45RA-PC7, CD197-PE, CD95-FITC, CD278-APC, HLA-DR-ECD from Beckman Coulter, Marseille, France). Moreover, detection of circulating $\mathrm{CD}_{163}{ }^{+}$monocytes was determined by using CD14-PE and CD163-Vioblue (Myltenyi Biotec, Bergisch Gladbach, Germany). 


\subsection{Immunofluorescence}

The multiplex immunofluorescence assay was performed on the Ventana Discovery ULTRA automated slides Stainer (Ventana, Tucson, AZ, USA). This technology uses sequential application of unmodified primary antibodies with, among each, a specific heat deactivation maintaining tissue and antigen epitope integrity and avoiding cross reactivity between reagents. After dewax and pretreatment, FFPE (Formalin-Fixed ParaffinEmbedded) slides were incubated with primary antibody CD16 (clone SP175, Ventana Medical Systems, Mannheim, Germany), CD8 (clone C8/144B, Agilent, DAKO, Glostrup, Danemark), Granzyme B (clone GRB7, Agilent, DAKO, Glostrup, Danemark), ICOS (clone D1K2T, Cell Signaling Technologies, Leiden, The Netherlands), CD86 (clone E2/G8P, Cell Signalling Technologies, Leiden, The Netherlands) CD163 (clone E2/G8P, Cell Signalling Technologies, Leiden, The Netherlands) and CD14 (clone EPR3653, Roche Diagnostic, Mannheim, Germany). Primary antibodies were visualized using the OmniMap-HRP (Horse radish peroxidase conjugated anti-rabbit, anti-mouse), Amplification anti-HQ HRP Multimer secondary systems and tyramide-conjugated fluorophore kits FAM, Red610, Rhodamin6G and Cy5 (Ventana, Tucson, AZ, USA). Counterstain was performed using Hematoxylin and Bluid Reagent (Ventana, Tucson, AZ, USA). Multiplex Immunofluorescence stained slides were scanned using a P250 Panoramic digital scanner from 3D Histech (Budapest, Hungary) with appropriate fluorophores used (Rhodamin6G, RED610, FAM, Cy5). Immunofluorescence image quantifications were performed using indicaLabs HALO Imaging Analysis software (PerkinElmer, Villepinte, France).

\subsection{Preparation of Human Monocyte-Derived Macrophages (MDMs)}

Peripheral blood mononuclear cells (PBMCs) were obtained from healthy blood donors (Etablissement Français du Sang, EFS, France). Written informed consents were obtained from the donors under EFS contract number 21/PVNT/TOU/IPBS01/2009-0052. According to articles L1243-4 and R1243-61 of the French Public Health Code, the contract was approved by the French Ministry of Science and Technology (agreement number AC 2009-921) (Paris, France).

Human Monocytes were isolated from PBMCs using negative selection Monocyte Isolation Kit II (Macs Miltenyi), as recommended by the manufacturer's protocol. Monocytes were cultured at $37^{\circ} \mathrm{C}$ and $5 \% \mathrm{CO}_{2}$ in Macrophage-Serum Free Media (SFM, Gibco, Scotland, UK) supplemented with L-glutamine (Invitrogen, Bleiswijk, The Netherlands) and penicillin/streptomycin (PS, Invitrogen, Bleiswijk, The Netherlands). Isolated monocytes were differentiated to pro-tumoral macrophages over three days by M-CSF (Macs Miltenyi, $200 \mathrm{UI} / \mathrm{mL}$ ) and IL-10 (Macs Miltenyi, $50 \mathrm{UI} / \mathrm{mL}$ ) stimulation.

\subsection{Opsonization and Co-Culture of Ovarian Carcinoma Tumor Cell Lines with Human MDMs}

Human ovarian cancer cell lines were labeled with the murlentamab anti-AMHRII antibody (3C23K or GM102 ${ }^{\circledR}, 10 \mu \mathrm{g} / \mathrm{mL}$ ), 3C23K-FCKO (an isotypic control, $10 \mu \mathrm{g} / \mathrm{mL}$ ) or $3 \mathrm{C} 23 \mathrm{~K}-\mathrm{CHO}$ (a normal fucose form, $10 \mu \mathrm{g} / \mathrm{mL}$ ) at $4{ }^{\circ} \mathrm{C}$ during $1 \mathrm{~h}$. Opsonized tumor cells were then resuspended in Dulbecco's modified Eagle's medium (DMEM, Gibco, Scotland, UK), supplemented with L-glutamine, PS and 10\% heat-inactivated fetal calf serum (FCS, Sigma, Salisbuty, UK) and added to the differentiated human macrophages at a 1:1 ratio.

In some experiments, anti-AMHRII antibodies were used in combination with an anti-PD-1 antibody, pembrolizumab (Selleck Chemicals, Selleck, USA) at $10 \mu \mathrm{g} / \mathrm{mL}$. This antibody was added every day in the co-culture until T cells cytometry analysis.

\subsection{Evaluation of Ovarian Carcinoma Tumor Cell Number}

SKOV3-R2 ${ }^{+}$or COV434-R2 ${ }^{+}$cells were stained with the CellTrace ${ }^{\mathrm{TM}}$ Violet Cell Proliferation kit (Molecular Probes ${ }^{\mathrm{TM}}$, Life technology, Bleiswijk, The Netherlands), opsonized with anti-AMHRII antibodies (3C23K, 3C23K-FcKO or 3C23K-CHO) and added to cultures of differentiated human macrophages at a 1:1 ratio. At different timepoint of the co-culture (Days 3, 4 and 5 according to the experimental protocol represented in 
Figure S2), SKOV3-R2 ${ }^{+}$cell number was evaluated by detecting fluorescently labeled cells by flow cytometry.

\subsection{Antibody-Dependent Cell-Mediated Cytotoxicity (ADCC) Assay}

After opsonization with the different anti-AMHRII antibodies, target SKOV3-R2 ${ }^{+}$cells were loaded with BATDA (Bis-acetoxymethyl-2,2' $: 6^{\prime}, 2^{\prime \prime}$-terpyridine-6, $6^{\prime \prime}$-dicarboxylate), resuspended in DMEM (Gibco, Scotland, UK) supplemented with L-glutamine, PS, and $10 \%$ heat-inactivated FCS and added tothe effector cells (human macrophages) at a 1:1 ratio, at $37^{\circ} \mathrm{C}$ for $4 \mathrm{~h}$.

ADCC was measured by using the DELFIA EuTDA-based cytotoxicity assay (PerkinElmer, Waltham, MA, USA). After $4 \mathrm{~h}$ of the co-incubation of target and effector cells, supernatants were then incubated with $\mathrm{Eu}^{3+}$ solution and fluorescence was measured (Envision, PerkinElmer, Waltham, MA, USA). Data were normalized to maximal (target cells with triton) and minimal (effector cells alone) lysis and fit to a sigmoidal dose-response model.

\subsection{Phenotypic Characterization of Human MDMs}

Receptor expression (CD32, CD64, CD80, TLR2, CD163, CD36 and CD206) was evaluated by flow cytometry at the membrane of differentiated human macrophages after 3 days of co-culture with opsonized SKOV3-R2 ${ }^{+}$tumor cells. Receptors were detected using CD32-PE-Vio770, CD64-PerCP-Vio700, CD80-PE, CD282 (TLR2)-APC, CD163-PE, CD36-PE and CD206-APC (Miltenyi, Bergisch Gladbach, Germany) and were compared with an appropriate isotype control.

A population of 10,000 cells was analyzed for each data point. Dead cells (positive cells) were removed from the analysis after labeling with Viobility Fixable Dye (Miltenyi, Bergisch Gladbach, Germany). Analyses were gated on CD14 or Cd11b positive cells. All analyses were performed using a BD Fortessa flow cytometer with the Diva software. The gating strategies are presented in the Figure S6.

\subsection{Production of Cytokines and Chemokines}

Cytokines (IL1 $\beta$, IL12, TNF $\alpha$, IL6, IFN $\gamma$, IL23 and IL10) and chemokines (CCL2, CCL4, CCL5, CXCL9 and CXCL10) release was quantified in the supernatant after 3 days of coculture between differentiated human macrophages and opsonized SKOV3-R2 ${ }^{+}$tumor cells and 4 days after $\mathrm{T}$ cell addition according to the manufacturer's instructions (AlphaLISA, PerkinElmer, Waltham, MA, USA).

\subsection{Human $T$ Cell Isolation}

Human T cells were isolated from PBMCs using negative selection Pan T Cell Isolation Kit (Macs Miltenyi, Bergisch Gladbach, Germany) and activated ( $\mathrm{T}$ cell activation/Expansion kit, Macs Miltenyi, Bergisch Gladbach, Germany) as recommended by the manufacturer's protocol. T cells were resuspended in RPMI 1640 Medium (Gibco, Scotland, UK) supplemented with L-glutamine, PS, and 10\% heat-inactivated FCS and added in the co-culture wells with human macrophages and opsonized SKOV3-R2 ${ }^{+}$tumor cells at a $1: 8$ ratio for 4 days.

\subsection{T Cell Polarization and Activation}

To profile lymphocyte populations, cells were labeled with the following antibodies: CD45-VioGreen, CD3-APCVio770, CD183 (CXCR3)-APC, CD25-PE, CD8-PEVio770 and CD4-ViobrightFITC (Macs Miltenyi, Bergisch Gladbach, Germany). A population of 10,000 cells was analyzed for each data point. All analyses were performed using a BD Fortessa flow cytometer with the Diva software. The gating strategies are presented in the Figure S7. 


\subsection{Secretory Profile of M2-Like Macrophages in the Presence of Murlentamab}

M2-like macrophages were generated in vitro by culturing $\mathrm{CD} 14^{+}$monocytes, isolated from healthy donors (Etablissement Français du Sang, Toulouse, France; EFS), in the presence of complete culture medium containing $50 \mathrm{ng} / \mathrm{mL}$ recombinant human M-CSF for 4 days, as previously described [56]. In parallel, anti-AMHRII antibodies were prepared in sterile and apyrogen PBS (10 mM, pH = 7.4; Lonza, Basel, Switzerland) and adsorbed for $24 \mathrm{~h}$ at $4{ }^{\circ} \mathrm{C}$ to 24 -well cell culture plates $(10 \mu \mathrm{g} / \mathrm{mL}, 1 \mathrm{~mL} /$ well $)$, as previously described. M-CSF-differentiated macrophages were cultured for 3 more days in antibody-coated plates $\left(10^{6}\right.$ cells/well). Cells were then stimulated or not for $24 \mathrm{~h}$ with $100 \mathrm{ng} / \mathrm{mL}$ LPS (Sigma-Aldrich, Salisbuty, UK), in the absence of the antibody, because, as previously reported, human macrophages have to be stimulated to reveal their phenotype [29]. The expression of CD16, CD64, and CD163 surface molecules was evaluated with, respectively, anti-CD16 (clone 20G8; BD Biosciences, Erembodegem, Belgium), anti-CD64 (clone 10.1; BD Biosciences, Erembodegem, Belgium) and anti-CD163 (clone 215927; R\&D Systems, Abingdon, UK) by flow cytometry using a FACSCanto II flow cytometer (BD Biosciences, Erembodegem, Belgium) and analyzed with the FlowJo software. IL1 $\beta, 6$, TNF $\alpha$, IL10 and IL12 in the culture supernatants of $24 \mathrm{~h}$ stimulated cells were quantified by ELISA (Diaclone, Besançon, France).

\subsection{In Vivo Experiment}

All procedures were reviewed and approved by the local ethics committee (Comite d'Ethique pour 1'Expérimentation Animale Genevois, ethic code: A740169; CELEAG). The experiments were carried out with four-week-old NOG (NOD/Shi-scid/IL-2R $\gamma$ null) immunodeficient mice ${ }^{\circledR}$ (Taconic, Doussard, France) engrafted with cord blood-derived $\mathrm{CD} 4^{+}$hematopoietic stem and progenitor cells (French Blood Bank, Annemasse, France) two days after chemical myeloablative treatment. Engraftment consisted in intravenous injection of $\mathrm{CD}_{4} 4^{+}$cells. Fourteen weeks after cell injection, engraftment level was monitored with the analysis of human $\mathrm{CD} 45^{+}$cells among total blood mouse and human leukocytes by flow cytometry (Attune, Gibco, Thermo Fisher scientific, Waltham, MA, USA). At the thirteenth week, macrophage/monocyte cell populations were enhanced in humanized NOG mice by receiving a treatment based on the transient expression of human cytokines GM-CSF/IL-3/IL4. Based on human $\mathrm{CD} 45^{+}$analysis, mice were randomized for tumor grafting.

Tumor cells, COV434-R2 ${ }^{+}$, were cultured as described previously. After washing steps, cells were suspended in PBS-EDTA (1mM), Matrigel (1:1) and $100 \mu \mathrm{L}$ of cell suspension $\left(10 \times 10^{6}\right.$ cells $)$ were injected subcutaneously in the right flank of mice. Day 0 was defined as the day of the cell inoculation. The presence of PD-L1 in cells was confirmed before engraftment by flow cytometry (data not shown).

At day 35, when tumors reached an average volume of $60-80 \mathrm{~mm}^{3}$, animals were randomized into 4 therapeutic groups of 8 mice based on their humanization rate, their number of myeloid cells (CD11b-positive cells) and their tumor volume. Murlentamab was diluted in PBS then administrated intraperitoneally, twice a week, for 4 weeks. The control groups were treated with PBS (vehicle) with the same schedule of administration as murlentamab. Pembrolizumab (MelonePharma, Dalian, China) was administrated per os at $50 \mathrm{mg} / \mathrm{kg}$ in water with $5 \%$ dextrose every day for 4 weeks. The fourth group was treated with a combo of pembrolizumab and murlentamab administrated as described above for monotherapy. Tumor growth was evaluated by measuring with a caliper two perpendicular tumor diameters twice a week. Tumor volume and tumor growth inhibition (TGI) were calculated according to the standard method [57]. In vivo toxicity was assessed as follows: weights of individual mice were measured every three days. Variation of mice weight as compared to their initial weight and means per group were calculated. A treatment was considered toxic and was stopped once a bodyweight loss of $15 \%$ was observed, and animals were sacrificed if body weight loss persisted for three consecutive 
days. Treatment was also stopped when an individual mouse had a body weight loss of $20 \%$ or more.

Some PD markers were followed during in vivo experiments. The blood of each mouse was collected from the retro-orbital sinus on EDTA-coated tubes (Microvette 100 MCVT100-EDTA, Sarstedt, Marnay, France) before tumor engraftment (Day 0) and at sacrifice. A total of $100 \mu \mathrm{L}$ of whole blood was stained with antibodies and lysed with red blood cell lysis buffer, washed with PBS and suspended in PBS before acquisition on the Attune Nxt Flow cytometer (Gibco, Thermo Fisher scientific, Waltham, MA, USA). In this study, the detection of hCD86 and hCD163 was performed by using, respectively, anti-CD86 Alexa-700 antibody (BD Biosciences, Erembodegem, Belgium) and anti-CD163 APC antibody (Miltenyi, Bergisch Gladbach, Germany).

\subsection{Flow Cytometry Antibodies}

Flow cytometry antibodies used were shown in Table 1.

\subsection{Statistical Analysis}

For each experiment, the data were subjected to one-way ANOVA analysis followed by the means multiple comparison method of Dunnett or Tukey. $p<0.05$ was considered as the level of statistical significance.

\section{Conclusions}

This study is the first to detail the mode of action of murlentamab, a low-fucose antibody, from its antigen binding to the tumor cell lysis via a cascade of activation of macrophage and lymphocytes in a sole in vitro study/model/schema integrating all the partners. Moreover, signs of activation of innate and adaptative immune response were confirmed in patients treated with murlentamab. These results open new perspectives in terms of combined treatment by associating murlentamab with checkpoint inhibitors or other immuno-modulators as many agents currently under clinical investigation.

\section{Patents}

Patent WO 2018/219956 A1 resulted from the work reported in this manuscript.

Supplementary Materials: The following are available online at https:/ / www.mdpi.com/article/ 10.3390/cancers13081845/s1, Figure S1: C101 and C201 clinical study design, Figure S2: Experimental protocol of the in vitro co-culture system. SKOV3 human ovarian tumor cells modified to strongly express AMHRII (SKOV3-R2 ${ }^{+}$) were opsonized with murlentamab (also called 3C23K, a low-fucose anti-AMHRII antibody), 3C23K-FcKO (a control antibody mutated in the constant part) or 3C23K-CHO (3C23K normal fucose form) at $10 \mathrm{ug} / \mathrm{mL}$. These opsonized tumor cells were then co-cultured with naïve unstimulated human MDMs (M0) or M-CSF/IL-10-treated human MDMs used to mimic tumor-associated macrophage (TAMs) found in the tumor microenvironment. For some experiments, autologous human T cells were added in the co-culture wells. For combo therapy experiments, pembrolizumab $(10 \mathrm{ug} / \mathrm{mL})$ was added in the culture everyday until the end of the experiment, Figure S3: Secretory profile of macrophages cultured with murlentamab. Macrophages were generated by culturing monocytes for 4 days in the presence of M-CSF. These M2-like macrophage were then cultured for 3 days in the absence (None) or presence of immobilized $3 \mathrm{C} 23 \mathrm{~K}-\mathrm{FcKO}$ or murlentamab. Pro- (TNF $\alpha$ and IL6) and anti-inflammatory (IL10) cytokines were quantified in the culture supernatants of LPS-stimulated cells. Results are expressed in $\mathrm{ng} / \mathrm{mL}$ (boxplots, $n=4) .{ }^{*} p<0.05 ;{ }^{* *} p<0.01 ;{ }^{* * *} p<0.001 ;{ }^{* * *} p<0.0001 . p$ values were determined using one-way ANOVA analysis followed by Dunnett's multiple comparisons test, Figure S4: Murlentamab opsonization of SKOV3-R2 ${ }^{+}$orients naïve macrophages and reprograms TAMs towards an M1-like profile in the presence of T cells. SKOV3-R2 ${ }^{+}$ovarian tumor cells were labeled with different $3 \mathrm{C} 23 \mathrm{~K}$ antibodies (3C23K-FcKO control, 3C23K-CHO normally fucosylated or murlentamab the low fucosylated form) and cultured in the presence of human monocyte-derived macrophages from healthy donors unstimulated (M0) or stimulated with M-CSF and IL-10 (TAMs). After 3 days of co-culture, activated T cells coming from the same healthy donor were added in the culture well for 4 more days. 
The release of cytokines (IL12, IFN $\gamma$, IL6, IL23 and IL10) and chemokines (CCL4, CCL5, CXCL9 and CXCL10) was determined by AlphaLISA. Data shown (boxplots) are the results from three different experiments (performed with three different healthy donors). ${ }^{*} p<0.05 ;{ }^{* *} p<0.01 ;{ }^{* *} p<0.001$; **** $p<0.0001$. $p$ values were determined using one-way ANOVA analysis followed by Dunnett's multiple comparisons test, Figure S5: Murlentamab opsonization of COV434-R2 ${ }^{+}$activates an effective anti-tumor $\mathrm{T}$ cell immune response. COV434-R2 ${ }^{+}$ovarian tumor cells were labeled with different $3 \mathrm{C} 23 \mathrm{~K}$ antibodies (3C23K-FcKO control or murlentamab the low fucosylated form) and cultured in the presence of human monocyte-derived macrophages from healthy donors unstimulated (M0) or stimulated with M-CSF and IL-10 (TAMs). After 3 days of co-culture, activated T cells coming from the same healthy donor were added in the culture well for 4 more days. (A) The CD4 $4^{+}$Th1/Th2 polarization profile and (B) the activation of $\mathrm{T} \mathrm{CD} 8^{+}$cells were determined by flow cytometry after four days of co-culture. Data shown (mean \pm SEM) are the results from three different experiments (performed with one healthy donors). ${ }^{*} p<0.05$; ${ }^{* *} p<0.01$; ${ }^{* * *} p<0.001$; ${ }^{* * *} p<0.0001 . p$ values were determined using one-way ANOVA analysis followed by Dunnett's multiple comparisons test, Figure S6: Gating strategies for the analysis of M1/M2 surface receptors at the membrane of human monocyte-derived macrophages. Forward scatter (FSC) and side scatter (SSC) parameters were used to identify single, viable cells and to eliminate any debris, dead cells and clumps or doublets. Cells were also stained with a viability dye to make sure to exclude all dead cells from the analysis. CD11bFITC, CD14-APCVio770, CD32-PEVio770, CD64-PerCPVio770, CD80-PE, CD282-APC, CD163-PE, CD206-APC and CD36-PEVio770 antibodies were used in different staining panels to evaluate the proportions of human monocyte-derived macrophages expressing the CD32, CD64, CD80, CD282, CD163, CD206 and CD36 surface markers as represented in the gating strategies, Figure S7: Gating strategies for the analysis of T cells. Forward scatter (FSC) and side scatter (SSC) parameters were used to identify single, viable cells and to eliminate any debris, dead cells and clumps or doublets. Cells were also stained with a viability dye to make sure to exclude all dead cells from the analysis. CD45-VioGreen, CD3-APCVio770, CD8-PEVio770, CD4-Viobright FITC, CD25-PE and CD183-APC antibodies were used to evaluate the proportions of $\mathrm{CD}^{+} \mathrm{CD} 183^{+}, \mathrm{CD}^{+} \mathrm{CD}^{2} 5^{-} \mathrm{CD} 183^{-}, \mathrm{CD} 4^{+}$ $\mathrm{CD} 25$ high, $\mathrm{CD}^{+} \mathrm{CD} 25^{-} \mathrm{CD} 183^{+} \mathrm{T}$ cells as represented in the gating strategies.

Author Contributions: Conceptualization, A.C. and J.-M.B.; methodology, M.S., T.A., M.P., A.C., G.N., L.C. and P.J.; validation, M.S., M.P., A.C., B.J., F.L. and P.J.; formal analysis, M.S., T.A., A.C., O.D., G.N. and L.P.; original draft preparation, M.P., M.S., A.C., O.D. and J.-M.B.; writing-review and editing, M.P., M.S., J.-M.B. and A.C.; supervision, I.T.-F., J.-F.P., B.P. and A.C. All authors have read and agreed to the published version of the manuscript.

Funding: This research received no external funding.

Institutional Review Board Statement: The clinical studies, C101 and C201, were conducted according to the guidelines of the Declaration of Helsinki. C101 study ( $\mathrm{N}^{\circ}$ EudraCT 2015-004252-22) was approved by the Ethic Committee of Jules Bordet, Belgium (22/03/2016), CPP Ile de France III, France (02/05/2016) and of URA Royal Marsden Hospital, UK (30/01/2018). C201 study (N EudraCT 2018-000627-13) was approved by Ethic Committee of UZ Leuven, Belgium (25/07/2018) and of FN Motol, Czech Republic (23/05/2018). For the in vivo study, all procedures were reviewed and approved by the local ethic committee (Comité d'Ethique pour l'Expérimentation Animale Genevois; approval number APAFIS@9218-2007031311022568v3).

Informed Consent Statement: Informed consent was obtained from all subjects involved in the study.

Data Availability Statement: All data relevant to the study are included in the article or uploaded as supplementary information.

Acknowledgments: We thank Alexia Zakaroff-Girard and Elodie Riant (TRI imaging platform, IFR150/I2MC) for flow cytometry technical assistance.

Conflicts of Interest: The authors declare no conflict of interest.

\section{References}

1. Baarends, W.M.; van Helmond, M.J.; Post, M.; van der Schoot, P.J.; Hoogerbrugge, J.W.; de Winter, J.P.; Uilenbroek, J.T.; Karels, B.; Wilming, L.G.; Meijers, J.H. A Novel Member of the Transmembrane Serine/Threonine Kinase Receptor Family Is Specifically Expressed in the Gonads and in Mesenchymal Cells Adjacent to the Müllerian Duct. Dev. Camb. Engl. 1994, 120, 189-197. 
2. di Clemente, N.; Wilson, C.; Faure, E.; Boussin, L.; Carmillo, P.; Tizard, R.; Picard, J.Y.; Vigier, B.; Josso, N.; Cate, R. Cloning, Expression, and Alternative Splicing of the Receptor for Anti-Müllerian Hormone. Mol. Endocrinol. 1994, 8, 1006-1020. [CrossRef] [PubMed]

3. Jamin, S.P.; Arango, N.A.; Mishina, Y.; Hanks, M.C.; Behringer, R.R. Genetic Studies of the AMH/MIS Signaling Pathway for Müllerian Duct Regression. Mol. Cell. Endocrinol. 2003, 211, 15-19. [CrossRef]

4. Visser, J.A.; Schipper, I.; Laven, J.S.E.; Themmen, A.P.N. Anti-Müllerian Hormone: An Ovarian Reserve Marker in Primary Ovarian Insufficiency. Nat. Rev. Endocrinol. 2012, 8, 331-341. [CrossRef]

5. Sriraman, V.; Niu, E.; Matias, J.R.; Donahoe, P.K.; MacLaughlin, D.T.; Hardy, M.P.; Lee, M.M. Müllerian Inhibiting Substance Inhibits Testosterone Synthesis in Adult Rats. J. Androl. 2001, 22, 750-758.

6. Bakkum-Gamez, J.N.; Aletti, G.; Lewis, K.A.; Keeney, G.L.; Thomas, B.M.; Navarro-Teulon, I.; Cliby, W.A. Müllerian Inhibiting Substance Type II Receptor (MISIIR): A Novel, Tissue-Specific Target Expressed by Gynecologic Cancers. Gynecol. Oncol. 2008, 108, 141-148. [CrossRef]

7. Song, J.Y.; Chen, K.Y.; Kim, S.Y.; Kim, M.R.; Ryu, K.S.; Cha, J.H.; Kang, C.S.; MacLaughlin, D.T.; Kim, J.H. The Expression of Müllerian Inhibiting Substance/Anti-Müllerian Hormone Type II Receptor Protein and MRNA in Benign, Borderline and Malignant Ovarian Neoplasia. Int. J. Oncol. 2009, 34, 1583-1591. [CrossRef]

8. Kersual, N.; Garambois, V.; Chardès, T.; Pouget, J.-P.; Salhi, I.; Bascoul-Mollevi, C.; Bibeau, F.; Busson, M.; Vié, H.; Clémenceau, B.; et al. The Human Müllerian Inhibiting Substance Type II Receptor as Immunotherapy Target for Ovarian Cancer: Validation Using the MAb 12G4. MABS 2014, 6, 1314-1326. [CrossRef]

9. Pépin, D.; Sosulski, A.; Zhang, L.; Wang, D.; Vathipadiekal, V.; Hendren, K.; Coletti, C.M.; Yu, A.; Castro, C.M.; Birrer, M.J.; et al. AAV9 Delivering a Modified Human Mullerian Inhibiting Substance as a Gene Therapy in Patient-Derived Xenografts of Ovarian Cancer. Proc. Natl. Acad. Sci. USA 2015, 112, E4418-E4427. [CrossRef]

10. Anttonen, M.; Färkkilä, A.; Tauriala, H.; Kauppinen, M.; MacLaughlin, D.T.; Unkila-Kallio, L.; Bützow, R.; Heikinheimo, M. Anti-Müllerian Hormone Inhibits Growth of AMH Type II Receptor-Positive Human Ovarian Granulosa Cell Tumor Cells by Activating Apoptosis. Lab. Invest. 2011, 91, 1605-1614. [CrossRef] [PubMed]

11. Beck, T.N.; Korobeynikov, V.A.; Kudinov, A.E.; Georgopoulos, R.; Solanki, N.R.; Andrews-Hoke, M.; Kistner, T.M.; Pépin, D.; Donahoe, P.K.; Nicolas, E.; et al. Anti-Müllerian Hormone Signaling Regulates Epithelial Plasticity and Chemoresistance in Lung Cancer. Cell Rep. 2016, 16, 657-671. [CrossRef] [PubMed]

12. Barret, J.-M.M.; Meseure, D.; Bataillon, G.; Andersson, N.; Auguste, A.; Dubreuil, O.; Färkkilä, A.; Perrial, E.; Bossard, C.; Loison, E.; et al. Abstract 774: Anti-Müllerian Hormone Type II Receptor (AMHRII) Found Expressed in Human NonGynecological Solid Tumors, Suggesting Potential Broader Applications for Anti-AMHRII-Based Therapy. Cancer Res. 2018, 78, 774. [CrossRef]

13. Estupina, P.; Fontayne, A.; Barret, J.-M.; Kersual, N.; Dubreuil, O.; Blay, M.L.; Pichard, A.; Jarlier, M.; Pugnière, M.; Chauvin, M.; et al. The Anti-Tumor Efficacy of 3C23K, a Glyco-Engineered Humanized Anti-MISRII Antibody, in an Ovarian Cancer Model Is Mainly Mediated by Engagement of Immune Effector Cells. Oncotarget 2017, 8, 37061-37079. [CrossRef] [PubMed]

14. Bougherara, H.; Némati, F.; Nicolas, A.; Massonnet, G.; Pugnière, M.; Ngô, C.; Le Frère-Belda, M.-A.; Leary, A.; Alexandre, J.; Meseure, D.; et al. The Humanized Anti-Human AMHRII MAb 3C23K Exerts an Anti-Tumor Activity against Human Ovarian Cancer through Tumor-Associated Macrophages. Oncotarget 2017, 8, 99950-99965. [CrossRef] [PubMed]

15. Jefferis, R.; Lund, J.; Pound, J.D. IgG-Fc-Mediated Effector Functions: Molecular Definition of Interaction Sites for Effector Ligands and the Role of Glycosylation. Immunol. Rev. 1998, 163, 59-76. [CrossRef]

16. Shields, R.L.; Lai, J.; Keck, R.; O'Connell, L.Y.; Hong, K.; Meng, Y.G.; Weikert, S.H.A.; Presta, L.G. Lack of Fucose on Human IgG1 N-Linked Oligosaccharide Improves Binding to Human Fcgamma RIII and Antibody-Dependent Cellular Toxicity. J. Biol. Chem. 2002, 277, 26733-26740. [CrossRef] [PubMed]

17. Shinkawa, T.; Nakamura, K.; Yamane, N.; Shoji-Hosaka, E.; Kanda, Y.; Sakurada, M.; Uchida, K.; Anazawa, H.; Satoh, M.; Yamasaki, M.; et al. The Absence of Fucose but Not the Presence of Galactose or Bisecting N-Acetylglucosamine of Human IgG1 Complex-Type Oligosaccharides Shows the Critical Role of Enhancing Antibody-Dependent Cellular Cytotoxicity. J. Biol. Chem. 2003, 278, 3466-3473. [CrossRef]

18. Herter, S.; Birk, M.C.; Klein, C.; Gerdes, C.; Umana, P.; Bacac, M. Glycoengineering of Therapeutic Antibodies Enhances Monocyte/Macrophage-Mediated Phagocytosis and Cytotoxicity. J. Immunol. 2014. [CrossRef] [PubMed]

19. Stephens, D.M. Venetoclax and Obinutuzumab for Frontline Treatment of Chronic Lymphocytic Leukemia. Blood 2019, 134, 1691-1696. [CrossRef]

20. O'Nions, J.; Townsend, W. The Role of Obinutuzumab in the Management of Follicular Lymphoma. Future Oncol. Lond. Engl. 2019, 15, 3565-3578. [CrossRef]

21. Paz-Ares, L.G.; Gomez-Roca, C.; Delord, J.-P.; Cervantes, A.; Markman, B.; Corral, J.; Soria, J.-C.; Bergé, Y.; Roda, D.; Russell-Yarde, F.; et al. Phase I Pharmacokinetic and Pharmacodynamic Dose-Escalation Study of RG7160 (GA201), the First Glycoengineered Monoclonal Antibody against the Epidermal Growth Factor Receptor, in Patients with Advanced Solid Tumors. J. Clin. Oncol. Off. J. Am. Soc. Clin. Oncol. 2011, 29, 3783-3790. [CrossRef]

22. Delord, J.-P.; Tabernero, J.; García-Carbonero, R.; Cervantes, A.; Gomez-Roca, C.; Bergé, Y.; Capdevila, J.; Paz-Ares, L.; Roda, D.; Delmar, P.; et al. Open-Label, Multicentre Expansion Cohort to Evaluate Imgatuzumab in Pre-Treated Patients with KRAS-Mutant Advanced Colorectal Carcinoma. Eur. J. Cancer 2014, 50, 496-505. [CrossRef] 
23. Temam, S.; Spicer, J.; Farzaneh, F.; Soria, J.C.; Oppenheim, D.; McGurk, M.; Hollebecque, A.; Sarini, J.; Hussain, K.; Soehrman Brossard, S.; et al. An Exploratory, Open-Label, Randomized, Multicenter Study to Investigate the Pharmacodynamics of a Glycoengineered Antibody (Imgatuzumab) and Cetuximab in Patients with Operable Head and Neck Squamous Cell Carcinoma. Ann. Oncol. 2017, 28, 2827-2835. [CrossRef] [PubMed]

24. Nimmerjahn, F.; Ravetch, J.V. Fcgamma Receptors as Regulators of Immune Responses. Nat. Rev. Immunol. 2008, 8, 34-47. [CrossRef]

25. De Henau, O.; Rausch, M.; Winkler, D.; Campesato, L.F.; Liu, C.; Cymerman, D.H.; Budhu, S.; Ghosh, A.; Pink, M.; Tchaicha, J.; et al. Overcoming Resistance to Checkpoint Blockade Therapy by Targeting PI3K $\gamma$ in Myeloid Cells. Nature 2016, 539, 443-447. [CrossRef] [PubMed]

26. Villanueva, M.T. Macrophages Steal the Show. Nat. Rev. Drug Discov. 2017, 16, 455. [CrossRef]

27. Tan, B.; Shi, X.; Zhang, J.; Qin, J.; Zhang, N.; Ren, H.; Qian, M.; Siwko, S.; Carmon, K.; Liu, Q.; et al. Inhibition of Rspo-Lgr4 Facilitates Checkpoint Blockade Therapy by Switching Macrophage Polarization. Cancer Res. 2018, 78, 4929-4942. [CrossRef]

28. Le Naour, A.; Prat, M.; Thibault, B.; Mével, R.; Lemaitre, L.; Leray, H.; Joubert, M.-V.; Coulson, K.; Golzio, M.; Lefevre, L.; et al. Tumor Cells Educate Mesenchymal Stromal Cells to Release Chemoprotective and Immunomodulatory Factors. J. Mol. Cell Biol. 2020, 12, 202-215. [CrossRef]

29. Duluc, D.; Delneste, Y.; Tan, F.; Moles, M.-P.; Grimaud, L.; Lenoir, J.; Preisser, L.; Anegon, I.; Catala, L.; Ifrah, N.; et al. TumorAssociated Leukemia Inhibitory Factor and IL-6 Skew Monocyte Differentiation into Tumor-Associated Macrophage-like Cells. Blood 2007, 110, 4319-4330. [CrossRef] [PubMed]

30. Wu, J.-Y.; Huang, T.-W.; Hsieh, Y.-T.; Wang, Y.-F.; Yen, C.-C.; Lee, G.-L.; Yeh, C.-C.; Peng, Y.-J.; Kuo, Y.-Y.; Wen, H.-T.; et al. Cancer-Derived Succinate Promotes Macrophage Polarization and Cancer Metastasis via Succinate Receptor. Mol. Cell 2020, 77, 213-227.e5. [CrossRef] [PubMed]

31. Wang, X.; Luo, G.; Zhang, K.; Cao, J.; Huang, C.; Jiang, T.; Liu, B.; Su, L.; Qiu, Z. Hypoxic Tumor-Derived Exosomal MiR301a Mediates M2 Macrophage Polarization via PTEN/PI3K $\gamma$ to Promote Pancreatic Cancer Metastasis. Cancer Res. 2018, 78, 4586-4598. [CrossRef] [PubMed]

32. Cassetta, L.; Pollard, J.W. Targeting Macrophages: Therapeutic Approaches in Cancer. Nat. Rev. Drug Discov. 2018, 17, 887-904. [CrossRef]

33. Xue, J.; Schmidt, S.V.; Sander, J.; Draffehn, A.; Krebs, W.; Quester, I.; De Nardo, D.; Gohel, T.D.; Emde, M.; Schmidleithner, L.; et al. Transcriptome-Based Network Analysis Reveals a Spectrum Model of Human Macrophage Activation. Immunity 2014, 40, 274-288. [CrossRef] [PubMed]

34. Locati, M.; Curtale, G.; Mantovani, A. Diversity, Mechanisms, and Significance of Macrophage Plasticity. Annu. Rev. Pathol. Mech. Dis. 2020, 15, 123-147. [CrossRef]

35. Murray, P.J. Macrophage Polarization. Annu. Rev. Physiol. 2017, 79, 541-566. [CrossRef]

36. Mantovani, A.; Sica, A.; Sozzani, S.; Allavena, P.; Vecchi, A.; Locati, M. The Chemokine System in Diverse Forms of Macrophage Activation and Polarization. Trends Immunol. 2004, 25, 677-686. [CrossRef] [PubMed]

37. Laviron, M.; Boissonnas, A. Ontogeny of Tumor-Associated Macrophages. Front. Immunol. 2019, 10. [CrossRef]

38. Prat, M.; Le Naour, A.; Coulson, K.; Lemée, F.; Leray, H.; Jacquemin, G.; Rahabi, M.C.; Lemaitre, L.; Authier, H.; Ferron, G.; et al. Circulating CD14high CD16low Intermediate Blood Monocytes as a Biomarker of Ascites Immune Status and Ovarian Cancer Progression. J. Immunother. Cancer 2020, 8. [CrossRef] [PubMed]

39. Kistner, L.; Doll, D.; Holtorf, A.; Nitsche, U.; Janssen, K.-P. Interferon-Inducible CXC-Chemokines Are Crucial Immune Modulators and Survival Predictors in Colorectal Cancer. Oncotarget 2017, 8, 89998-90012. [CrossRef]

40. Skytthe, M.K.; Graversen, J.H.; Moestrup, S.K. Targeting of CD163+ Macrophages in Inflammatory and Malignant Diseases. Int. J. Mol. Sci. 2020, 21, 5497. [CrossRef]

41. Mallmann, M.; Schmidt, S.; Schultze, J. Macrophages in Human Cancer: Current and Future Aspects. Atlas Genet. Cytogenet. Oncol. Haematol. 2012. [CrossRef]

42. Heusinkveld, M.; van der Burg, S.H. Identification and Manipulation of Tumor Associated Macrophages in Human Cancers. J. Transl. Med. 2011, 9, 216. [CrossRef] [PubMed]

43. Niwa, R.; Shoji-Hosaka, E.; Sakurada, M.; Shinkawa, T.; Uchida, K.; Nakamura, K.; Matsushima, K.; Ueda, R.; Hanai, N.; Shitara, K. Defucosylated Chimeric Anti-CC Chemokine Receptor 4 IgG1 with Enhanced Antibody-Dependent Cellular Cytotoxicity Shows Potent Therapeutic Activity to T-Cell Leukemia and Lymphoma. Cancer Res. 2004, 64, 2127-2133. [CrossRef]

44. Horton, H.M.; Bernett, M.J.; Pong, E.; Peipp, M.; Karki, S.; Chu, S.Y.; Richards, J.O.; Vostiar, I.; Joyce, P.F.; Repp, R.; et al. Potent in Vitro and in Vivo Activity of an Fc-Engineered Anti-CD19 Monoclonal Antibody against Lymphoma and Leukemia. Cancer Res. 2008, 68, 8049-8057. [CrossRef] [PubMed]

45. Leary, A.; Aftimos, P.G.; Delord, J.-P.; Le Tourneau, C.; Ray-Coquard, I.L.; Jungels, C.; Varga, A.I.; Ricci, F.; Gomez-Roca, C.A.; Bataillon, G.; et al. A First-in-Human Study of Monoclonal Antibody GM102 in Patients with Anti-Mullerian-Hormone-Receptor II (AMHRII) Positive Gynecological Cancers. J. Clin. Oncol. 2018, 36, 5542. [CrossRef]

46. Van Cutsem, E.; Melichar, B.; Van den Eynde, M.; Prausova, J.; Geboes, K.; Dekervel, J.; Vitaskova, D.; Cuyper, A.D.; Linke, Z.; Acker, N.V.; et al. Phase 2 Study Results of Murlentamab, a Monoclonal Antibody Targeting the Anti-Mullerian-HormoneReceptor II (AMHRII), Acting through Tumor-Associated Macrophage Engagement in Advanced/Metastatic Colorectal Cancers. Ann. Oncol. 2019, 30, iv153-iv154. [CrossRef] 
47. Mantovani, A. Tumour-Associated Macrophages as Treatment Targets in Oncology. Clin. Oncol. 2017, 14, 399. [CrossRef]

48. Bournazos, S.; Gupta, A.; Ravetch, J.V. The Role of IgG Fc Receptors in Antibody-Dependent Enhancement. Nat. Rev. Immunol. 2020, 20, 633-643. [CrossRef]

49. Yeap, W.H.; Wong, K.L.; Shimasaki, N.; Teo, E.C.Y.; Quek, J.K.S.; Yong, H.X.; Diong, C.P.; Bertoletti, A.; Linn, Y.C.; Wong, S.C. CD16 Is Indispensable for Antibody-Dependent Cellular Cytotoxicity by Human Monocytes. Sci. Rep. 2016, 6, 34310. [CrossRef]

50. Ikeda, H.; Old, L.J.; Schreiber, R.D. The Roles of IFN $\gamma$ in Protection against Tumor Development and Cancer Immunoediting. Cytokine Growth Factor Rev. 2002, 13, 95-109. [CrossRef]

51. Tugues, S.; Burkhard, S.H.; Ohs, I.; Vrohlings, M.; Nussbaum, K.; vom Berg, J.; Kulig, P.; Becher, B. New Insights into IL-12-Mediated Tumor Suppression. Cell Death Differ. 2015, 22, 237-246. [CrossRef] [PubMed]

52. Uchida, J.; Hamaguchi, Y.; Oliver, J.A.; Ravetch, J.V.; Poe, J.C.; Haas, K.M.; Tedder, T.F. The Innate Mononuclear Phagocyte Network Depletes B Lymphocytes through Fc Receptor-Dependent Mechanisms during Anti-CD20 Antibody Immunotherapy. J. Exp. Med. 2004, 199, 1659-1669. [CrossRef] [PubMed]

53. Srivastava, R.M.; Lee, S.C.; Andrade Filho, P.A.; Lord, C.A.; Jie, H.-B.; Davidson, H.C.; López-Albaitero, A.; Gibson, S.P.; Gooding, W.E.; Ferrone, S.; et al. Cetuximab-Activated Natural Killer and Dendritic Cells Collaborate to Trigger Tumor AntigenSpecific T-Cell Immunity in Head and Neck Cancer Patients. Clin. Cancer Res. Off. J. Am. Assoc. Cancer Res. 2013, 19, 1858-1872. [CrossRef] [PubMed]

54. House, I.G.; Savas, P.; Lai, J.; Chen, A.X.Y.; Oliver, A.J.; Teo, Z.L.; Todd, K.L.; Henderson, M.A.; Giuffrida, L.; Petley, E.V.; et al. Macrophage-Derived CXCL9 and CXCL10 Are Required for Antitumor Immune Responses Following Immune Checkpoint Blockade. Clin. Cancer Res. 2020, 26, 487-504. [CrossRef]

55. Zhang, H.; Vollmer, M.; De Geyter, M.; Litzistorf, Y.; Ladewig, A.; Dürrenberger, M.; Guggenheim, R.; Miny, P.; Holzgreve, W.; De Geyter, C. Characterization of an Immortalized Human Granulosa Cell Line (COV434). Mol. Hum. Reprod. 2000, 6, 146-153. [CrossRef]

56. Jeannin, P.; Paolini, L.; Adam, C.; Delneste, Y. The Roles of CSFs on the Functional Polarization of Tumor-Associated Macrophages. FEBS J. 2018, 285, 680-699. [CrossRef]

57. Némati, F.; Sastre-Garau, X.; Laurent, C.; Couturier, J.; Mariani, P.; Desjardins, L.; Piperno-Neumann, S.; Lantz, O.; Asselain, B.; Plancher, C.; et al. Establishment and Characterization of a Panel of Human Uveal Melanoma Xenografts Derived from Primary and/or Metastatic Tumors. Clin. Cancer Res. Off. J. Am. Assoc. Cancer Res. 2010, 16, 2352-2362. [CrossRef] [PubMed] 